(

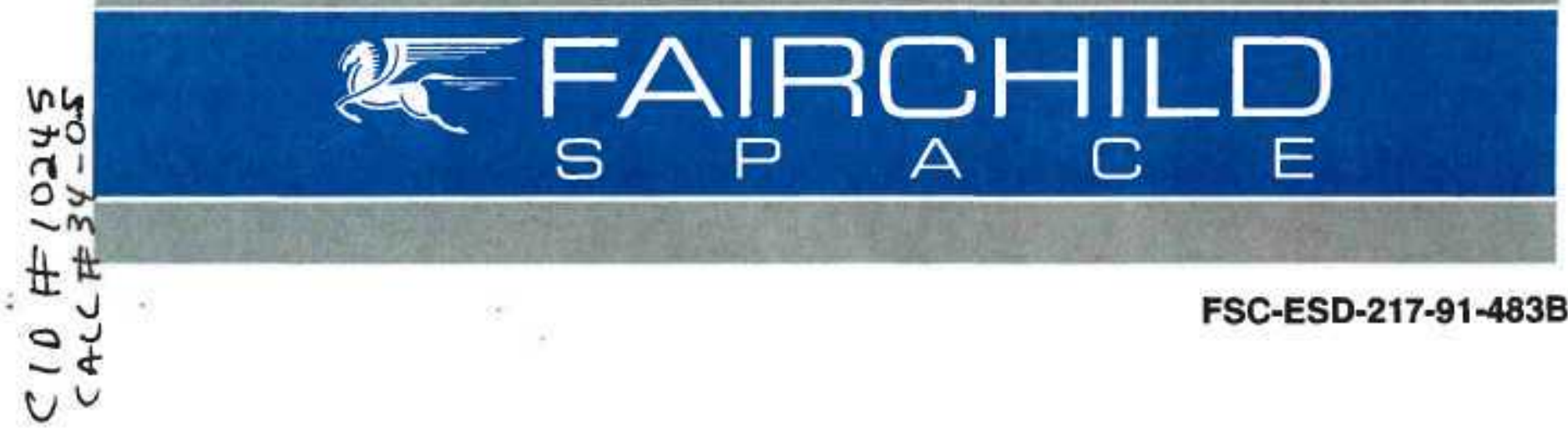

\title{
DESIGN, ANALYSIS, AND SPACECRAFT INTEGRATION OF RTGS FOR CRAF AND CASSINI MISSIONS
}

Author \& Principal Investigator:

A. Schock
Contributors:

T. Or

H. Noravian

JULY 10, 1991 



\section{DESIGN, ANALYSIE, AND SPACECRAFT INTEGRATION OF RTGs FOR CRAF AND CASSINI MISSIONS}

Author \& Principal Investigator:

A. Schock
Contributors:

T. Or

H. Noravian

JULY 10, 1991 



\begin{abstract}
This report consists of two parts. Part I describes the development of novel analytical methods needed to predict the BOM performance and the subsequent performance degradation of the mutually obstructing ATGs mounted near each other on the Mariner/Mark-2 spacecraft for the CRAF, Cassini, and subsequent missions. Part II applies those methods to the two missions, presents the resultant power predictions, and discusses their programmatic impications.

The results indicate that JPL's original power demand goals could have been met with two standard GPHS RTGs for each mission. However, JPL subsequently raised both the power demand protile and the duration for both missions, to the point where two standard RTGs could no longer provide the desired power margin. Each mission can be satisfied by adding a third RTG, and in the case of the Cassini mission the use of three RTGs appears to be unavoidable. In the case of the CRAF mission, there appeared to be a possibility that modest moditications of the RTGs' design and/or operating scheme may meet the missions' power demand without the addition of a third RTG. The potential saving in cost and schedule pressure prompted Fairchild to undertake a study of various obvious and not-so-obvious stratagems, either singly or in combination, to determine whether they would make it possible to meet the specified power demand with two RTGs.
\end{abstract}

The various stratagems investigated by Fairchild, and their effect on performance are presented. The analytical results indicate that a combination of relatively modest RTG modifications could come close to meeting the then-specified CRAF power demand goals. However, since even with the modifications the two RTGs did not provide sufficient margin for anticipated further growth in power demand, the JPL project team ultimately decided to use three RTGs for the CRAF mission also. This offered the advantage of minimizing the need for load switching to reduce the power demand peaks. The report documents the various power enhancement schemes and their computed effectiveness for possible future applications, and predicts the power output of the three obstructed RTGs over the life of each mission.

\section{INTRODUCTION}

The Jet Propulsion Laboratory (JPL) is in the process of designing spacecraft for NASA's upcoming CRAF and Cassini missions [1]. The CRAF (Comet Rendezvous and Asteroid Flyby) spacecraft, planned for a 1996 launch, will fly by at least one asteroid, and rendezvous with a comet, where it will conduct scientific observations for more than two years. The Cassini spacecraft, scheduled for a late-1995 launch, will also fly by at least one asteroid, fly by Jupiter, orbit the planet Saturn, repeatedly fly by a number of Saturn's moons, and send an instrumented probe, called the Huygens probe, into the atmosphere of Saturn's moon Titan.
Both missions will be launched by unmanned Titan-4/Centaur-G' boosters, and both are part of a series which will use a new generation of cost-effective modular spacecraft (Mariner/Mark 2) that can easily be modified to accomplish a variety of missions to comets and asteroids and to the outer solar system.

Because of their great distance from the sun, RTG (Radioisotope Thermoelectric Generator) power supplies have been baselined for both missions. Since the planned schedules do not allow sufficient time for development and flight qualification of new RTG designs, both missions will use generators that are essentially identical to the GPHS-RTGs flown on the recently launched Galileo and Ulysses missions. JPL's original plans called for two such RTGs on each mission. Their construction will be under the direction of the U.S. Department of Energy's Office of Special Applications (DOE/OSA), which commissioned Fairchild Space Company to conduct RTG studies in support of JPL's design efforts.

The location and orientation of the two RTGs on the spacecraft are functions of numerous, often conflicting, design constraints. The CRAF/Cassini baseline design that JPL initially asked Fairchild to study is depicted in Figure 1.

Figure 1. CRAF/CASSINI Spacecraft, Initial Design

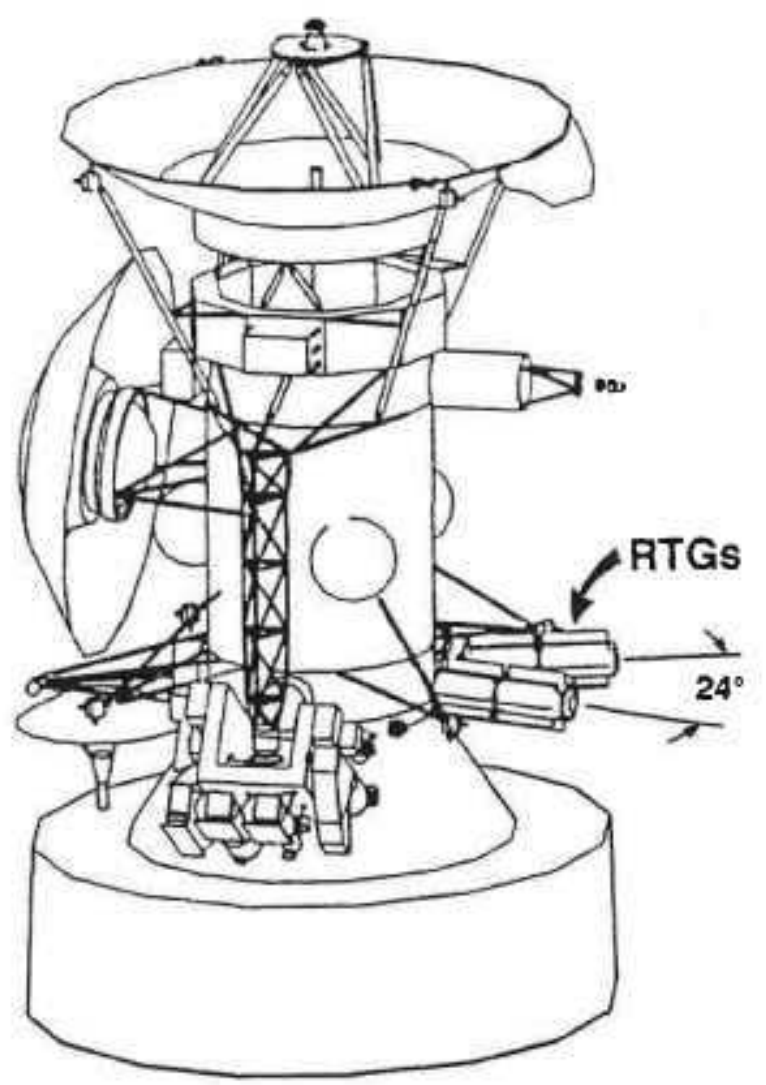


Figure 1 shows the two RTGs cantilevered from the cylindrical spacecraft like radial spokes, with a separation angie of 24 degrees between them. Such proximity leads to mutual obstruction of the two RTGs' heat rejection paths. This obstruction can result in significant axial and circumferential variation of the cold-junction temperatures and the electrical performance of the thermoelectric couples in each RTG.

To assess the effect of mutual obstructions on the RTGs' output power, Fairchild personnel were asked to analyze the baseline configuration shown in Figure 1 , as well as some alternative configurations. Because of the unconventional problem, this required the development of novel analysis methods and computer codes, which are described in this paper. The analytical results reported here served as inputs for JPL's spacecraft design decisions.

\section{RTG DESIGN DESCRIPTION}

Figure 2 shows a cutaway view of the GPHS-RTG $[2,3,4]$ that will be used on the CAAF and Cassini missions. Each 1.15 m-long RTG contains an axial stack of 18 General Purpose Heat Source modules [5], which radiate their heat to a surrounding cylindrical array of $\mathbf{5 7 6}$ thermoelectric unicouples arranged in 36 layers of 16 couples.

\section{Figure 2. GPHS RTG}

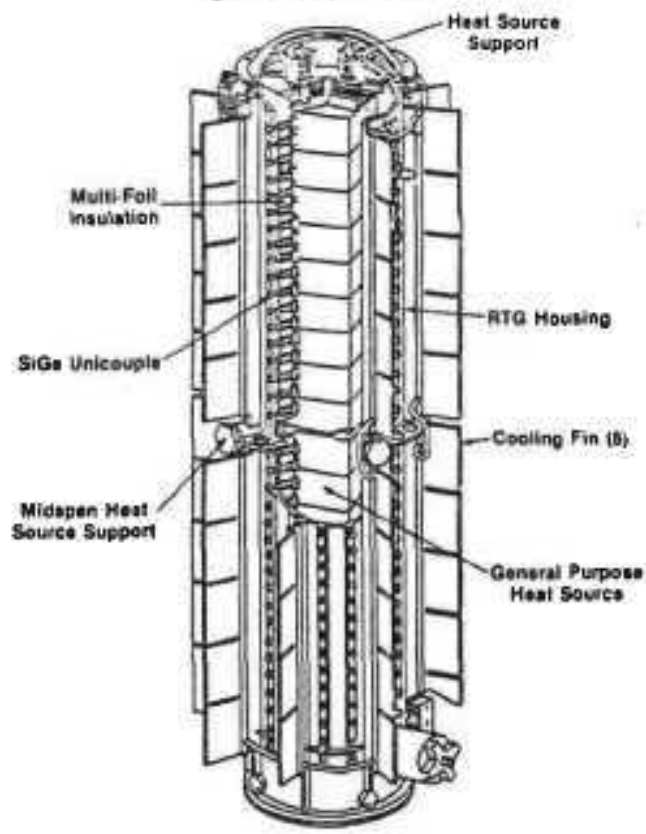

As shown in Figure 3, each unicouple contains a thermoelectric $n$ - and p-leg. These are electrically connected at their hot ends by a hot-shoe, which serves to collect the heat radiated by the centrally located heat source stack and concentrate it at the thermoelectric legs. The cold end of each leg is series-and parallel-connected to adjoining couples to form the RTG's electrical network. The couples' cold ends are bolted to the RTG housing, to which they reject their waste heat. The RTG's waste heat is dissipated by radiation from its housing and its eight equispaced radiator fins, as shown in Figure 2.

Figure 3. Unicouple

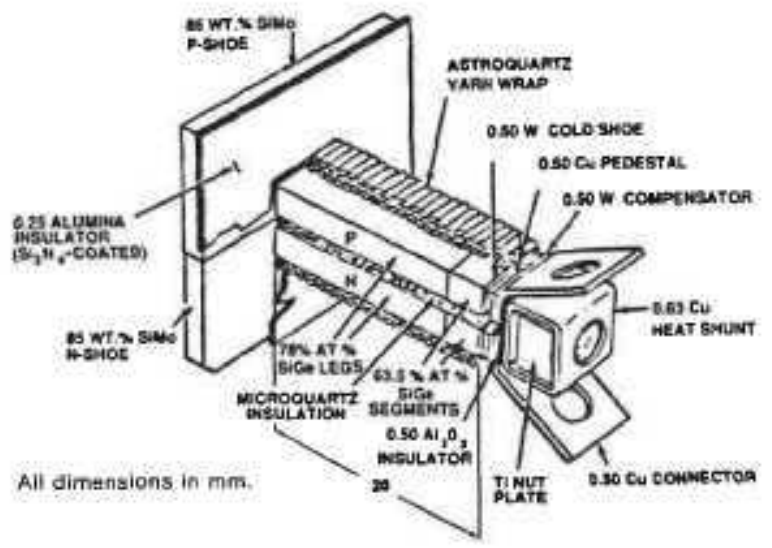

The series-parallel network of the GPHS-RTG is shown in Figure 4, which depicts a rolled-out schematic of the cylindrical array. The electrical network consists of two parallel branches. Each branch contains 144 series-connected groups of two parallel couples. The rather tortuous current path shown is designed to minimize the RTG's self-induced magnetic field.

Figure 4. Current-Path Through GPHS RTG

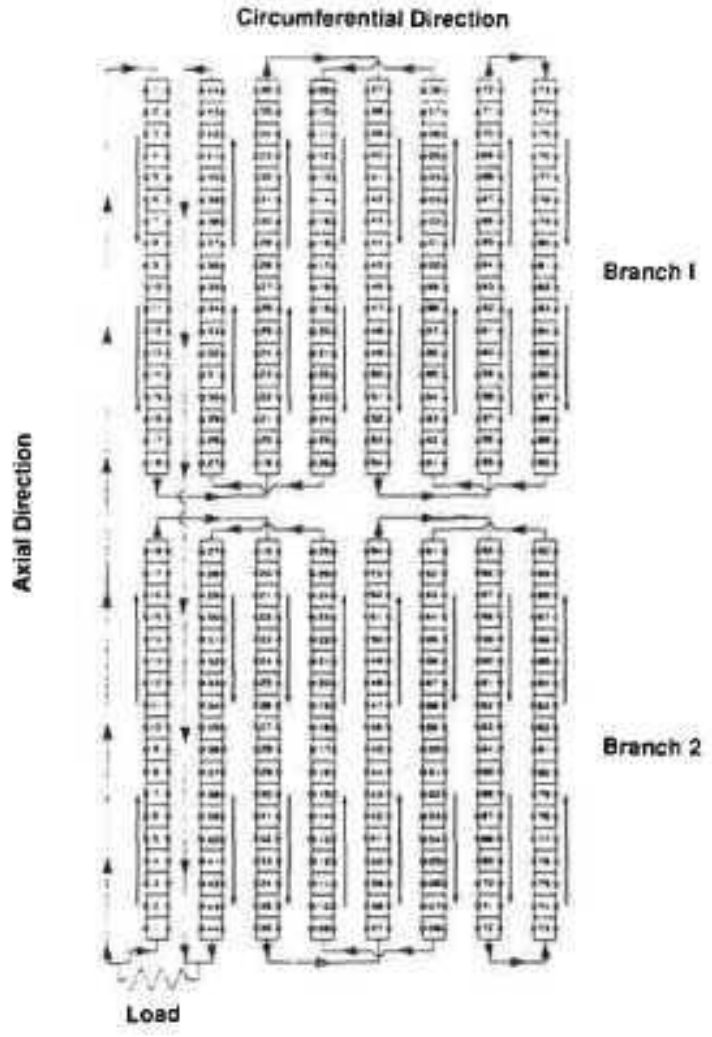




\section{ANALYSIS}

The close proximity of the two RTGs on the CAAF/Cassini spacecraft, as illustrated by the design shown in Figure 1, can result in significant mutual blockage of their heat rejection paths. Such blockage would result in circumferential variation of the RTG's housing and cold-junction temperatures. Determining the effect of that temperature variation on the RTGs' power output requires a very detailed and careful analysis, because we are looking for relatively small differences between large numbers, and because even small differences can be quite significant if the mission is power-constrained (as are the CRAF and Cassini missions).

Previous RTG analyses usually made the simplifying assumption that all of the thermoelectric couples in a generator's series-parallel network operate at the same hot-and coid-junction temperatures and at the same current and voltage. For unobstructed RTGs, such a simplified analysis is a useful initial design tool, since it permits closed-form solutions for the optimum area ratio $A_{n} / A_{p}$ of the thermoelectric $n$ - and $p$-legs and for the optimum output voltage. For these optimized parameters, it yieids simple expressions for the maximum material efficiency of the thermoelectric couples, and for the required RTG design parameters $[6,7,8]$.

But the above simplifying assumptions can introduce significant errors even for unobstructed RTGs, because all RTGs have appreciable axial temperature variations due to unavoidabie end losses by radiation and by conduction through the heat source support structure. A more exact analysis, which accounted for the axial temperature variations in a Martian RTG, was reported last year $[7,9]$. But that analysis still assumed that the RTG has an axisymmetric view of space and the Martian ground. and therefore no circumferential temperature variation.

The present paper develops a Fairchild-generated methodology and generalized computer code for analyzing the performance of arbitrarily obstructed RTGs with both axial and circumferential temperature. voltage, and current variations, and applies that methodology to the specific example of the CRAF/Cassini design depicted in Figure 1.

\section{COUPLED THERMAL AND ELECTRICAL ANALYSIS}

Figure 5 presents the energy balance for a thermoelectric unicouple of leg length $L$, leg areas $A_{n}$ and $A_{p}$, operating between cold- and hot-junction temperatures $T_{1}$ and $T_{2}$. It gives the couple's thermal conductance $\mathbf{K}$, electric resistance $\mathbf{R}$, and open-circuit voltage $V_{0}$ in terms of the temperature-averaged thermal conductivity $\bar{k}$, electrical resistivity $\bar{\rho}$, and Seebeck coefficient $\bar{S}$ of the thermoelectric $n$ - and $p$ - materials. As shown, the heat input rate $Q_{h}$ at the couple's hot end and the heat rejection rate $\mathrm{Q}_{c}$ at its cold end each consists of four terms: normal heat conduction, Peitier effect, Ohmic dissipation, and Thomson effect. As can be seen, three of those four terms are current-dependent. Therefore, the thermal and electrical analyses cannot be pertormed separately, but must be conducted simultaneously and interactively.

Figure 5. Unicouple Energy Balance

$$
\begin{array}{ll}
\text { Open-Circuit Voltage: } & V_{0}=\int\left(S_{n}+S_{p}\right) d T \\
\text { Couple Conductance: } & K=\left[\bar{k}_{n} A_{n}+\bar{k}_{p} A_{p}\right] / L \\
\text { Couple Resistance: } \quad & R=\left[\bar{\rho}_{n} / A_{n}+\bar{\rho}_{p} / A_{p}\right] L
\end{array}
$$

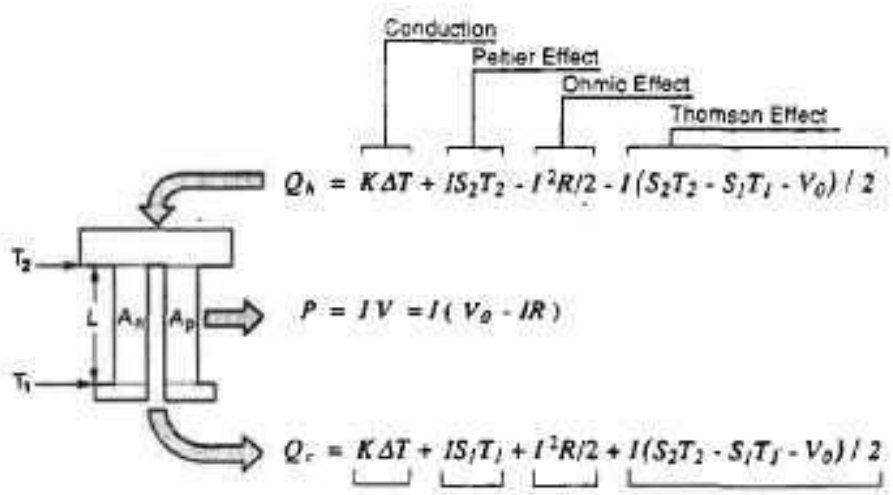

To analyze an RTG of a given design with an unsymmetrically obstructed heat rejection path, a detailed three-dimensional thermal model of the RTG and its environment must be constructed. The hot junction and cold junction of each thermoelectric element in the RTG are represented as discrete nodes. The model cannot be analyzed by means of a standard thermal analysis code, because the connectors between the couple's hot and cold junctions are not simple thermal conductors but include the current-dependent Peltier, Ohmic, and Thomson effects. The rate at which heat enters the connector's hot-end and leaves its cold-end are not equal, since part of the heat entering each couple is converted to electrical energy. That part must in effect be represented as a heat sink for each couple.

The electrical analysis is further complicated by the cons'raint that each RTG's thermoelectric couples are in general interconnected in a complex seriesparallel network, and that all couples grouped in parallel must operate at the same output voltage, and that all couple groups in series must produce the same current.

The RTG analysis methodology developed by Fairchild is generic, not just for the GPHS-RTG network shown in Figure 4. In general, the equivalent 
circuit of an RTG networik consists of B parallel branches, with each branch containing $G$ seriesconnected groups of $\mathbf{C}$ parallel couples. Each thermoelectric element in the RTG is designated by a branch number b, group number $\mathbf{g}$, and couple number c, where:

$$
\begin{aligned}
& 1 \leq \mathrm{b} \leq \mathrm{B}, \\
& 1 \leq \mathrm{g} \leq \mathrm{G}, \\
& 1 \leq \mathrm{C} \leq \mathrm{C} .
\end{aligned}
$$

For the case of the GPHS-RTG circuit depicted in Figure $4, B=2, G=144, C=2$, and the equivalent circuit of the generator is shown in Figure 6 .

Figure 6. Equivalent Circuit of GPHS RTG

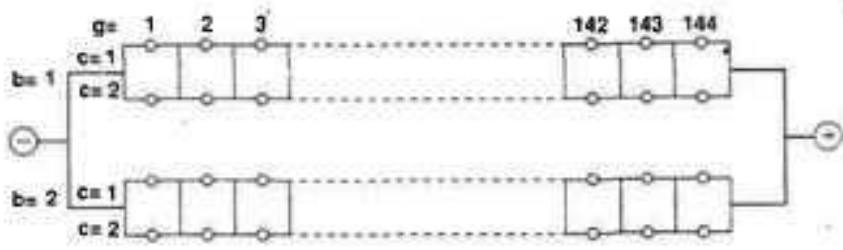

\section{ELECTRICAL ANALYSIS}

For each couple in the RTG, the difference between its open-circuit voltage $V_{0}(b, g, c)$ and its internal voltage drop $I(b, g, c) R(b, g, c)$ equals its output voltage $V(b, g, c)$. Since the couples in each group $b, g$ are connected in parallel, their output voltage must equal the group voltage $\mathrm{V}(\mathrm{b}, \mathrm{g})$.

$$
V_{0}(b, g, c)-I(b, g, c) R(b, g, c)=V(b, g, c)=V(b, g)
$$

Therefore, the current through couple $b, g, c$ is:

$$
I(b, g, c)=\left[V_{\alpha}(b, g, c) \cdot V(b, g)\right] / R(b, g, c) \text {. }
$$

The sum of each group's $\mathrm{C}$ couple currents equals the group current $\mathrm{I}(\mathrm{b}, \mathrm{g})$. Since all groups in each branch $\mathrm{b}$ are connected in series, this must equal the branch current I(b):

$$
\sum_{c=I}^{C}[V(b, g, c) / R(b, g, c)]-V(b, g) \sum_{c=I}^{C}[l / R(b, g, c)]=I(b, g)=I(b)
$$

Therefore, the voltage produced by group $b, g$, is:

$$
V(b, g)=\left\{\sum_{c=1}^{C}\left[V_{0}(b, g, c) / R(b, g, c)\right]-I(b)\right\} / \sum_{c=1}^{C}[1 / R(b, g, c)]
$$

The sum of the $G$ group voltages in each branch equals the branch voltage $V(b)$. Since the $B$ branches are connected in parallel, this also equals the RTG's output voltage $V_{\text {RTG: }}$

$$
\sum_{g=I}^{G}\left\{\sum_{c=1}^{c}\left[V_{0}(b, g, c) / R(b, g, c)\right] / \sum_{c=l}^{c}[1 / R(b, g, c)]\right\}-I(b) \sum_{g=I}^{G}\left\{1 / \sum_{c=I}^{C}[1 / R(b, g, c)]\right\}=V(b)=V_{R T G} .
$$

Solving for the branch current I(b) for each branch and summing the currents for the B parallel branches gives the RTG output current:

$$
I_{R T G}=\sum_{b=1}^{B}\left\{\left[\sum_{k=1}^{G}\left\{\sum_{c=1}^{C}\left[V_{O}(b, g, c) / R(b, g, c)\right] / \sum_{c=1}^{C}[1 / R(b, g, c)]\right\}-V_{R T G}\right] / \sum_{g=1}^{G}\left\{1 / \sum_{c=1}^{C}[1 / R(b, g, c)]\right\}\right\} .
$$

The above equation represents the current-voltage characteristic of the RTG network. It can be expressed in the condensed form

$$
I_{R T G}=I_{S C}-V_{R T G} / R_{R T G} .
$$

where $I_{s c}$ is the RTG's shert-circuit current, defined by

$$
I_{S C}=\sum_{b=1}^{B}\left[\sum_{g=1}^{G}\left\{\sum_{c=l}^{C}\left[V_{O}(b, g, c) / R(b, g, c)\right] / \sum_{c=1}^{C}[1 / R(b, g, c)]\right\} / \sum_{g=1}^{G}\left\{1 / \sum_{c=1}^{C}[1 / R(b, g, c)]\right\}\right],
$$

$\mathbf{R}_{\text {RTG }}$ is the RTG's internal resistance, defined by

$$
R_{R T G}=1 / \sum_{b=1}^{B}\left[1 / \sum_{g=1}^{G}\left\{1 / \sum_{c=1}^{C}[1 / R(b, g, c)]\right\}\right],
$$

and the product IsC R $_{\text {RTG }}$ is the RTG's open-circuit voltage. 


\section{ITERATIVE COMPUTATIONS}

For each iteration in the analysis, the code uses each couple's cold- and hot-junction temperatures $\mathbf{T}_{\mathbf{1}}$ and $T_{2}$ (from the preceding iteration) to compute its temperature-averaged properties $\bar{k}$ and $\bar{p}$, open-circuit voltage $v_{0}$, thermal conductance $K$ and electrical resistance $R$ :

$$
\begin{aligned}
& \bar{k}_{n}(b, 8, c)=\int_{T_{f(b, g, c)}}^{T_{2}(b, f, c)} k_{n}(T) d T /\left[T_{2}(b, g, c)-T_{1}(b, g, c)\right] \\
& \bar{k}_{p}(b, g, c)=\int_{T_{f(b, g, c)}}^{T_{y}(b, c, c)} k_{p}(T) d T /\left[T_{2}(b, g, c) \cdot T_{l}(b, 8, c)\right] \\
& \left.\bar{\rho}_{n}(b, g, c)=\int_{T_{j(b, g, c)}}^{T_{2,(b, k, c)}} \rho_{n}(T), T\right) d T / \bar{k}_{n}(b, g, c)\left[T_{2}(b, g, c)-T_{l}(b, g, c)\right] \\
& \bar{\rho}_{p}(b, g, c)=\int_{T_{1(b, 8, c)}}^{T_{2(b, 8, c)}} \rho_{p}(T) k_{p}(T) d T / \bar{k}_{p}(b, g, c)\left[T_{2}(b, g, c) \cdot T_{1}(b, g, c)\right] \\
& V_{o}(b, g, c)=\int_{T_{l}(b, 8, c)}^{T_{2}(b, g, c)}\left[S_{n}(T)+S_{p}(T)\right] d T \\
& K(b, g, c)=\left[\bar{k}_{n}(b, g, c) A_{n}+\bar{k}_{p}(b, g, c) A_{p}\right] / L \\
& R(b, g, c)=\left[\bar{\rho}_{m}(b, g, c) / A_{n}+\bar{\rho}_{p}(b, g, c) / A_{p}\right] L
\end{aligned}
$$

Using these values of $V_{0}, K$, and $R$ for each couple and the prescribed RTG voltage $V_{R T G}$, the code computes the branch current $h(b)$ for each of the $B$ branches,

$$
I(b)=\left[\sum_{g=1}^{G}\left\{\sum_{c=1}^{C}\left[V_{0}(b, g, c) / R(b, g, c)\right] / \sum_{c=l}^{C}[1 / R(b, g, c)]\right\}-V_{R T C}\right] / \sum_{g=1}^{G}\left\{1 / \sum_{c=1}^{C}[1 / R(b, 8, c)]\right\},
$$

the group voltage $V(b, g)$ for each of the $G$ groups in each branch,

$$
V(b, g)=\left\{\sum_{c=1}^{C}\left[V_{0}(b, g, c) / R(b, g, c)\right]-I(b)\right\} / \sum_{c=1}^{C}[1 / R(b, g, c)],
$$

and the couple current l(b,g,c) for each couple in the RTG,

$$
I(b, g, c)=\left[V_{O}(b, g, c) \cdot V(b, g)\right] / R(b, g, c) .
$$

The individual couple currents are then used to compute the hot-end heat input rate $Q_{H}(b, g, c$,$) and the cold-end$ heat rejection rate $Q_{c}(b, g, c)$ for each couple:

$$
\begin{aligned}
Q_{H}(b, g, c) & =K(b, g, c)\left[T_{2}(b, g, c)-T_{I}(b, g, c)\right]-I^{2}(b, g, c) R(b, g, c) / 2 \\
& +I(b, g, c)\left[S_{2}(b, g, c) T_{2}(b, g, c)+S_{I}(b, g, c) T_{I}(b, g, c)+V_{O}(b, g, c)\right] / 2 . \\
Q_{c}(b, g, c) & =K(b, g, c)\left[T_{2}(b, g, c)-T_{1}(b, g, c)\right]+I^{2}(b, g, c) R(b, g, c) / 2 \\
& +I(b, g, c)\left[S_{2}(b, g, c) T_{2}(b, g, c)+S_{I}(b, g, c) T_{I}(b, g, c)-V_{0}(b, g, c)\right] / 2 .
\end{aligned}
$$

where $S_{1}$ and $S_{2}$ denote the Seebeck coefficients at the cold- and hot-junction of the couple.

The code inserts these heat flow rates for each couple in the RTG into the detailed thermal analysis model for the next iteration; and repeats the procedure until convergence is achieved. 


\section{CODE VALIDATION}

The code was first tested in the analysis of an unobstructed generator, with axiai temperature variation but no circumferential variation. It was validated by using it to analyze the pertormance of the electrically heated thermoelectric generator (ETG) that had been employed as the engineering test unit [10], a prototype of the GPHS-RTGs used for the Galileo mission. The reason for using the ETG instead of the RTG as a validation check for the code is that the ETG test measurements include the thermocouples' hotshoe temperatures, but - because of practical difficulties - the RTG measurements do not.

The analysis of the ETG's performance was based on thermoelectric properties of SiGe aged for one year to account for pre-test outgassing and processing. The temperature-dependent values of resistivity, conductivity, and Seebeck coefficient of the $\mathrm{n}$ - and $\mathrm{p}$-material used in the analysis are summarized in Table 1.

\begin{tabular}{|c|c|c|c|c|c|c|}
\hline \multirow[t]{2}{*}{$\begin{array}{l}\text { Temp } \\
{ }^{\circ} \mathrm{C}\end{array}$} & \multicolumn{2}{|c|}{$\begin{array}{l}\text { Seebeck } \\
\mu \mathrm{V} / \mathrm{K}\end{array}$} & \multicolumn{2}{|c|}{$\begin{array}{c}\text { Resistivity } \\
\text { m } \Omega, \mathrm{cm}\end{array}$} & \multicolumn{2}{|c|}{$\begin{array}{c}\text { Conductivity } \\
\mathrm{mw} / \mathrm{cm}, \mathrm{k}\end{array}$} \\
\hline & $\mathrm{N}$ & P & $N$ & P & $\mathrm{N}$ & p \\
\hline 0 & 90 & 114 & 0.79 & 0.91 & 51.4 & 58.4 \\
\hline 50 & 113 & 128 & 0.87 & 0.95 & 50.2 & 57.2 \\
\hline 100 & 135 & 140 & 0.95 & 1.01 & 49.0 & 56.3 \\
\hline 150 & 153 & 152 & 1.04 & 1.08 & 47.9 & 55.0 \\
\hline 200 & 168 & 163 & 1.13 & 1.15 & 46.8 & 54.1 \\
\hline 250 & 185 & 173 & 1.28 & I. 24 & 45.8 & 52.9 \\
\hline 300 & 215 & 183 & 1.72 & 1.32 & 44.7 & 51.7 \\
\hline 350 & 265 & 192 & 2.77 & 1.42 & 43.9 & 51.0 \\
\hline 400 & 304 & 202 & 3.81 & 1.53 & 43.1 & 49.9 \\
\hline 450 & 317 & 211 & 4.17 & 1.64 & 42.5 & 49.1 \\
\hline 500 & 317 & 220 & 4.02 & 1.74 & $4 i .8$ & 48.2 \\
\hline 550 & 312 & 229 & 3.75 & 1.87 & 41.5 & 47.5 \\
\hline 600 & 306 & 240 & 3.46 & 2.06 & 41.0 & 46.8 \\
\hline 650 & 298 & 256 & 3.13 & 2,38 & 40.8 & 46.2 \\
\hline 700 & 289 & 287 & 2.82 & 2.98 & 40.6 & 45.7 \\
\hline 750 & 260 & 320 & 2.53 & 4.49 & 40.6 & 45.3 \\
\hline 80 & 27 & 331 & 2.28 & 4.76 & 40.7 & 45.2 \\
\hline 85 & 26 & 330 & 2.09 & 4.51 & 40.9 & 45.4 \\
\hline 90 & & 323 & 1.95 & 4.17 & 41.4 & 46.0 \\
\hline & & 318 & 1.86 & 3.84 & 42.1 & 47.1 \\
\hline 100 & & 316 & 1.82 & 3.57 & 43,4 & 49.2 \\
\hline & & & 1.77 & & 45.6 & 52.2 \\
\hline & 2 & 314 & 1.75 & 3.10 & 46.9 & 54.3 \\
\hline
\end{tabular}

The analytical results were compared with the ETG test measurements. For the same RTG thermal power and the same average cold-junction temperature, the experimental measurements and the analytical results produced by the code were in very good agreement. The average hot-junction temperatures agreed within $5^{\circ} \mathrm{C}$ (986 versus 981$)$, and the electrical power outputs agreed within 1 watt $(296$ versus 297).
As a further check on the validity of the analytical model, it was used to compute the BOM performance of one of the Galileo flight RTGs (F4). For the same thermal power, it yielded an electrical output of 285.6 watts, compared to the measured output power of 287.7 watts in Earth orbit. This agreement is better than the estimated $1.2 \%$ telemetry error.

The good agreement between the analytical and experimental results for both the ETG and the RTG lends confidence to our use of the same model and assumptions for subsequent analyses of the initial RTG output at various thermal powers and external environments.

\section{ANALYSIS OF OBSTRUCTED CRAF/CASSINI RTGS}

The application of the code to the analysis of the obstructed CRAF/Cassini RTGs started with the construction of a 1912-node radiation-interchange analysis model. The model represented the housing and fins of the two GPHS-FTGs pictured in Figure 2 and the spacecraft shown in Figure 1. The ITAS (Integrated Thermal Analysis System) code [11], which accounts for the effect of mutual reflections, was used to compute over 102,000 radiation interchange factors between all surface nodes that are within each other's view. The computed radiation interchange factors were then inserted into a detailed thermal and electrical analysis model consisting of -2900 node points.

The coupled thermal and electrical analysis was carried out by means of the previously discussed computer code. The code was based on the SINDA thermal analysis program [12], modified by Fairchild to incorporate the current-dependent Peltier, Ohmic, and Thomson effects on thermocouple conductance and to represent the electrical power generation in each couple as an effective heat sink. In each iteration, the modified code computed each couple's heat input rate and heat rejection rate and inserted them into the thermal analysis for the next iteration. After the solution had homed in to prescribed convergence criteria, it was used to calculate the RTG's electrical output and efficiency.

To illustrate typical results, the converged BOM solution is summarized in Tables 2 through 5 for the configuration depicted in Figure 1 and a thermal power of 245 watts from each of the 18 heat source modules. The tables display the results for the flattened-out cylindrical array of 576 thermocouples in the RTG. 
Table 2 shows the axial and circumferential variation of the RTG's cold-junction temperatures. As can be seen these vary from $276^{\circ} \mathrm{C}$ for the least obstructed couple to $302^{\circ} \mathrm{C}$ for the most obstructed couple. The last column and last line of the table show the variation of the averaged coidjunction temperatures in the axial and circumferential directions, respectively. As shown, the average temperature is lowest near the ends of the RTG, and highest near the RTG's midplane. Circumferentially, the average temperature is lowest in Column 6, the outward-facing side of the RTG, and highest in Column 14, the RTG side facing the neighboring RTG.

Table 3 similarly shows the axial and circumferential variations of the baseline RTG's hot-junction temperatures. As can be seen, the couples' average hot-junction temperatures vary by $35^{\circ} \mathrm{C}$ in the axial direction, and show almost no variation in the circumferential direction. Thus, the obstruction by the neighboring RTG and by the spacecraft affects only the cold-junction temperatures, and produces only a negligible circumferential variation of the hot-junction temperatures. In addition to the hot-junction temperatures; the table shows the axial variation of the heat source surface temperatures. As shown by the table's left column, these vary from $1008^{\circ} \mathrm{C}$ at the upper outboard end of the heat source stack to $1042^{\circ} \mathrm{C}$ near the middle of the stack.

The consequent variation in the thermocouples' temperature-spans affects their electrical performance. The axial and circumferential variations of the couple voltages are displayed in Tabie 4, and those of the couple currents in Table 5 . The sixteen columns in these tables represent eight column pairs of parallel couples. Table 4 shows that all parailel couples have the same output voltage, ranging from 0.197 volt to 0.215 volt; and Table 5 shows that all couple pairs in each branch have the same combined output current, as demanded by the RTG's series-parallel network.

As can be seen, the network's two branches have respective output currents 5.14 and 5.16 amp, for a total output of 10.30 amps per RTG. The 144 series-connected couple groups in each branch produce 30.0 volts. Subtracting 0.34 volts for ohmic losses in the RTG's series leads leaves a net output of 29.66 volts and 309 watts per ATG. The average material efficiency of the couples is $7.90 \%$; the average couple efficiency (including the effect of contact resistances and electrode losses) is $7.55 \%$; and the net system efficiency (including the effect of heat losses through the thermal insulation and through the heat source support structure) is $7.01 \%$.
Table 2. Cold-Junction Temperatures $\left({ }^{\circ} \mathrm{C}\right)$

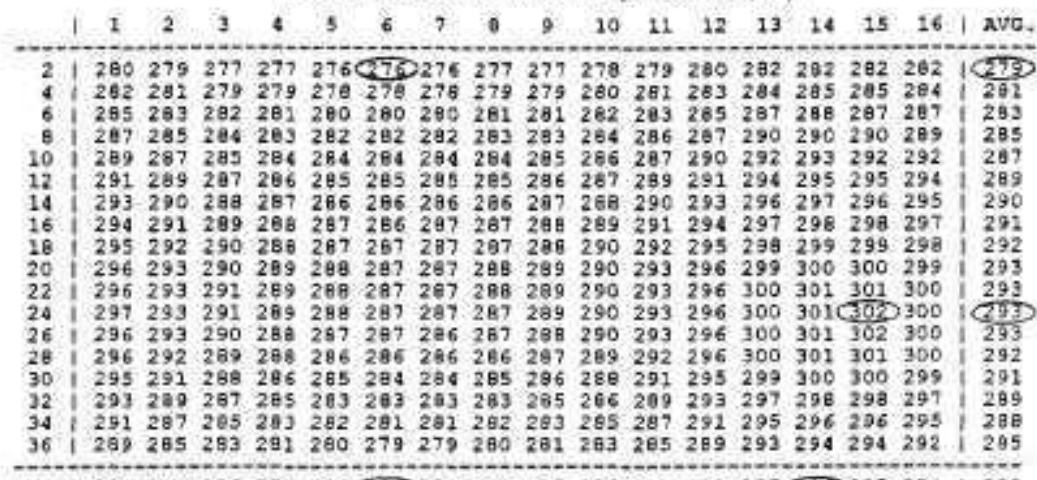

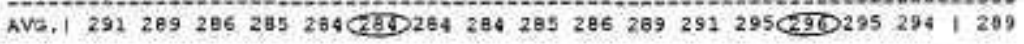

Table 3. Hot-Junction Temperatures $\left({ }^{\circ} \mathrm{C}\right)$

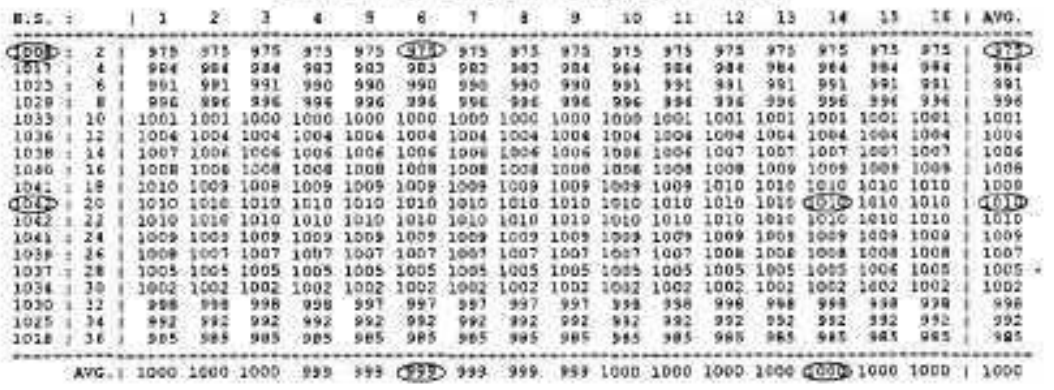

Table 4. Couple Voltage (mV)

\begin{tabular}{|c|c|c|c|c|c|c|c|c|c|c|c|c|c|c|c|c|c|}
\hline & 13 & 2 & I & 4 & ; & C & ? & E & 9 & 10 & 11 & 12 & 1) & 14 & 15 & 16 & AW \\
\hline & 19 & 199 & 200 & 200 & 201 & 201 & $a z$ & 201 & 200 & 200 & 110 & 149 & 199 & 199 & 198 & 298 & 199 \\
\hline 4 & & 203 & 204 & 206 & 205 & 209 & as & 205 & 204 & 204 & 03 & 203 & 201 & 201 & 202 & 202 & \\
\hline & 208 & 206 & 207 & 207 & 208 & 200 & 200 & 200 & 207 & 200 & 206 & 706 & 204 & 204 & 6 & 208 & 206 \\
\hline 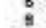 & 208 & 2006 & 210 & 210 & $2 \neq 0$ & 210 & 220 & 210 & 209 & 203 & 200 & 200 & 206 & 206 & 206 & 206 & 200 \\
\hline 18 & 210 & 210 & 21 & 211 & 212 & 212 & 2 & $\begin{array}{l}210 \\
212\end{array}$ & 221 & 211 & 8 & 209 & 207 & 207 & 207 & 207 & 210 \\
\hline $1:$ & 2 & 2110 & $\begin{array}{l}211 \\
212\end{array}$ & 211 & $\begin{array}{l}212 \\
223\end{array}$ & 212 & 21. & $\begin{array}{l}212 \\
213\end{array}$ & 212 & 212 & $\begin{array}{l}209 \\
210\end{array}$ & 21 & 205 & $2 \mathrm{CO}$ & 200 & 200 & 211 \\
\hline if & $2 i 2$ & 212 & 33 & 213 & 228 & 214 & 21 & $2 \pm 4$ & 213 & 213 & $\frac{2}{2}$ & 2 & 209 & 2009 & & 209 & $2 \mathrm{i2}$ \\
\hline & $\begin{array}{l}212 \\
212\end{array}$ & 212 & 234 & 214 & 213 & 21 & 21 & 215 & 214 & i1 & z: & 2. & 20 & 20 & 2 & 209 & 212 \\
\hline & & 212 & & & & & & & & & & & & & & & \\
\hline 20 & & 211 & 216 & 211 & 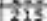 & 215 & 275 & 213 & 224 & $z$ & $-\frac{1}{21}$ & $\frac{10}{213}$ & $\frac{200}{20}$ & & 20 & सेग & 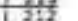 \\
\hline 22 & 212 & 211 & 214 & 214 & 215 & 21 & & 218 & 21 & & & & 200 & 20 & & 2 & $2 i$ \\
\hline 20 & 2 & 21 & 21 & 213 & 214 & 22 & 2. & 214 & 21 & 22 & 21 & 2. & 20 & 20 & & & $2 \mathrm{~s}$ \\
\hline 2 & 2 & ii & 2 & 212 & 21 & 2i & 2 & 214 & 21 & 2. & 21 & 2. & 20 & 20 & & 20 & 260 \\
\hline 26 & 2 & 30 & 2 & 211 & 213 & 21 & 2 & 21 & 21 & 2. & 205 & 20 & 205 & 2. & 20 & 2 & 209 \\
\hline & & 2 & 2 & i. & 2 & 2 & 2 & 21 & 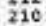 & 21 & & 2 & & 2 & 20 & 2 & 20 \\
\hline 92 & & & & 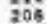 & & & & & 2 & 20 & & 2 & $i$ & 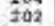 & & 2 & 20 \\
\hline 3 & 2 & 20 & 2 & & & & & & 20 & & & & & 30 & & 2 & 20 \\
\hline $\overrightarrow{3 i}$ & 200 & 200 & 201 & 203 & 204 & 204 & 20 & 204 & 201 & 203 & 200 & 200 & j97 & 297 & 197 & in & 201 \\
\hline & 06 & & 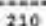 & 2 & 2 & 2 & 211 & 211 & 230 & 210 & 200 & 200 & 205 & 205 & 205 & 208 & 209 \\
\hline
\end{tabular}

Table 5. Couple Current (amp)

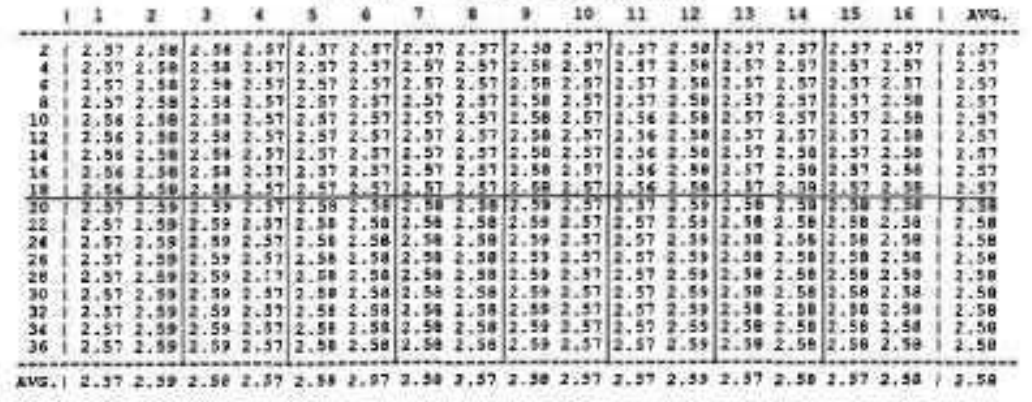

Equally detailed coupled thermal and electrical analyses were performed for thermal power levels ranging from 225 to 250 watts per heat source module, for both obstructed and unobstructed RTGs. The unobstructed units had axial temperature variations but no circumferential variation. The principal results for the mutually obstructed RTGs in the CRAF/Cassini baseline configuration (Figure 1) and the comparative results for an unobstructed RTG are displayed in Figures 7,8 , and 9. 
For a separation angle of 24 degrees between the two RTGs, Figures 7 and 8 respectively show the effect of the thermal power on the RTG power output and on the average hot-junction temperature. As can be seen, both relationships are essentially linear.

Figure 7. Effect of Thermal Power on BOM Electrical Power

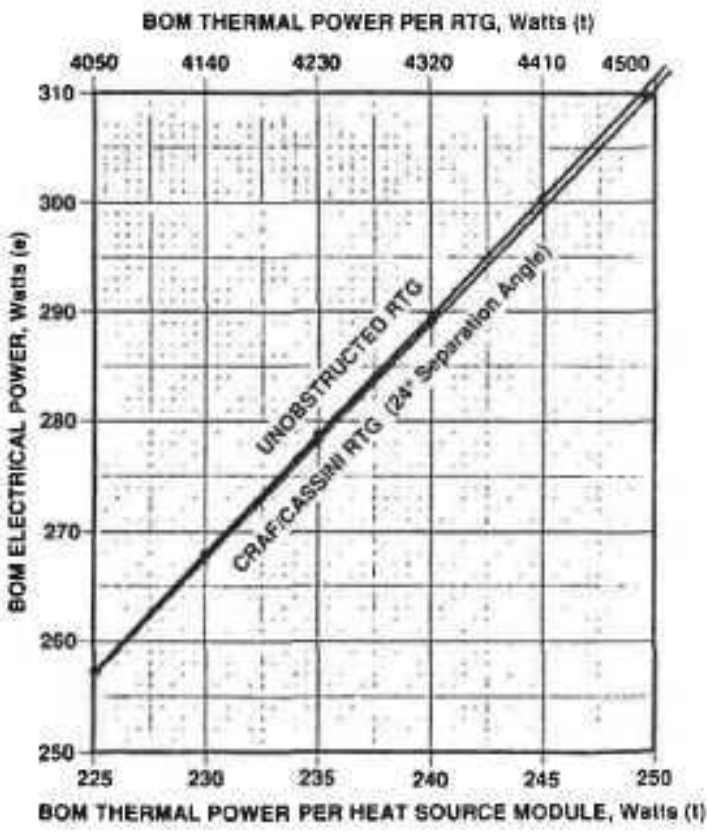

$\rightarrow \infty$

Figure 8. Effect of Thermal Power on Hot-Junction Temperature

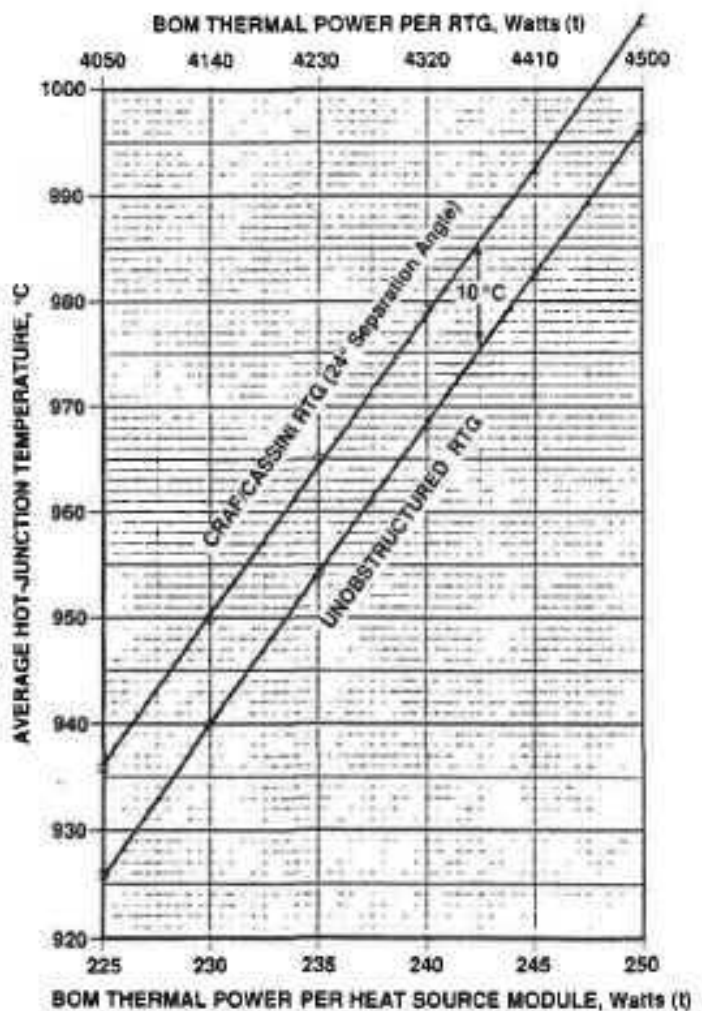

The two figures show that, for a given thermal power, the obstruction by the neighboring RTG has very little effect on the RTG's power output, but has a significant effect on its hot-junction temperature, which affects the degradation rate. Figure 9 presents crosspiots showing the relationship between maximum hotjunction temperatures and BOM power outputs for unobstructed RTGs and for mutually obstructed RTGs with separation angles of $16^{\circ}, 24^{\circ}$, and $35^{\circ}$.

Figure 9. Effect of Separation Angle $\Theta$ Between RTGs on Power-Versus-Temperature Characteristics

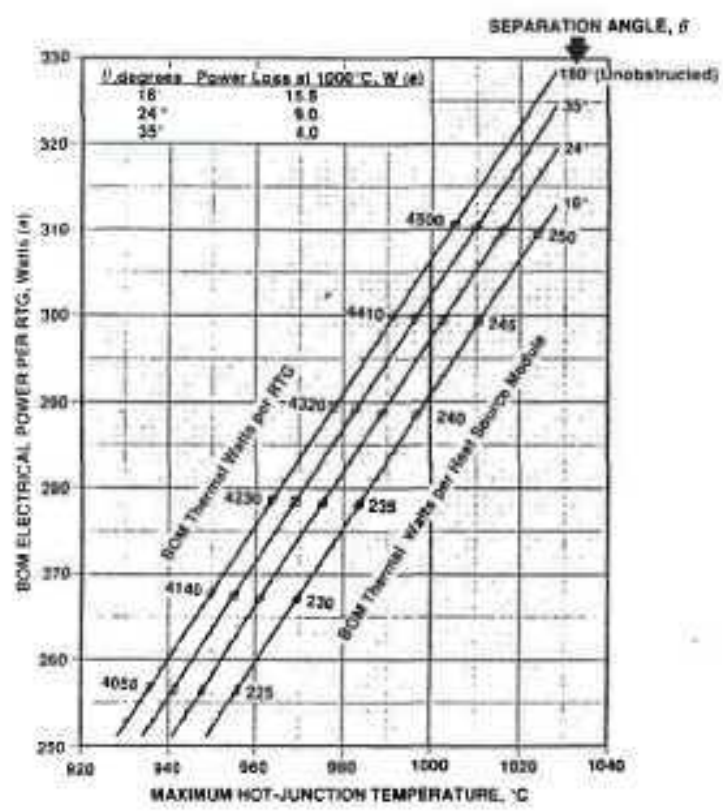

The performance of the obstructed and unobstructed RTGs should be compared for the same maximum hot-junction temperature, since that is what determines the ATG's degradation rate and lifetime. Previous SiGe flight units (LES 8/9, Voyager, Galileo) were designed for a maximum hot-junction temperature of $1000^{\circ} \mathrm{C}$. For that temperature, Figure 9 shows that the blockage by its neighbor reduces each RTG's power output by 15.5 watts $(5.1 \%)$ for a 16-degree separation angle, by 9.0 watts $(2.9 \%)$ for the 24-degree separation of JPL's original baseline design and by 4.0 watts $(1.3 \%)$ for the 35 -degree separation of JPL's revised spacecraft design. This demonstrates the sensitivity of power output to separation angle.

The 9-watt performance penalty for the 24-degree separation angle may not seem large. But both missions are severely power-constrained, and the JPL designers were unwilling to give up 18 watts from the two RTGs. Subsequently, the spacecraft designs were changed to a 35-degree separation angle, which results in much less power loss. 


\section{LONG-TERM PERFORMANCE DEGRADATION}

The system designer is interested not only in the RTGs' BOM power, but also in their power history throughout the mission. The principal factors that diminish an RTG's output power P with time $t$ are fuel decay and thermoelectric degradation. The combined effect of these factors can be represented by

$$
\frac{P}{P_{1}}=\left(\frac{P_{u}}{P_{1}}\right)\left(\frac{P}{P_{L}}\right) \text {. }
$$

where $P_{1}$ is the RTG's BOM power, the ratio $P_{U} / P_{1}$ is the effect of fuel decay alone (l.e.. just the effect of thermal power decrease, if there were no degradation), and the ratio $P / P_{u}$ is the effect of thermoelectric degradation for the RTG's predicted temperature history.

As shown in Figure 7 , in the range of practical interest the RTG's undegraded output power $P_{u}$ is a linear function of its thermal power $Q$ :

$$
P_{u}=P^{*}+\eta^{*} Q \text {. }
$$

The coefficients $\mathbf{P}^{*}$ and $\eta^{*}$ must be determined by the previously described detailed thermal and electrical analyses, taking account of the location, orientation, and mutual blockage of the RTGs on the spacecratt. For example, for two standard GPHS-RTGs separated by an angle of 35-degrees (JPL's revised baseline design), least-square analysis of the analytic results yields the curve-fit coefficients

$$
P^{*}=-218.0 \mathrm{w} \text {, and } \eta^{*}=0.1188 \text {. }
$$

Note that the coefficient $\eta^{\circ}$ is a differential efficiency, which is the sum of the actual efficiency $\eta$ and a term that reflects the fact that increasing $Q$ increases the temperature drop $\Delta T$ between hot- and cold-junctions, which in turn increases the efficiency

$$
\eta^{*} \equiv \frac{d P}{d Q}=\frac{d(Q \eta)}{d Q}=\eta+Q\left(\frac{\partial \eta}{\partial \Delta T)}\right)\left(\frac{\partial \Delta T)}{\partial Q}\right)
$$

The effect of fuel decay on the thermal power $Q$ at mission time $t$ is given by

$$
Q=Q_{1} \exp (-\lambda t) \text {. }
$$

where $Q_{1}$ is the BOM value of $Q$, and $\lambda$ is the $P u-238$ decay constant, which is related to its half-life $\tau(87.74$ years) by

$$
\lambda=(\ln 2) / \tau .
$$

Thus, the effect of fuel decay alone is given by

$$
\frac{P_{H}}{P_{l}}=1 \cdot \frac{l-e^{\cdot \lambda t}}{\left(P^{*} / \eta^{*} Q_{l}\right)+1}
$$

Predicting the thermoelectric performance degradation with time is generally a complex problem, since many different degradation mechanisms are at play. A detailed method for making such predictions is the DEGRA code developed by V. Raag [13], but that code does not account for the detailed effects of mutual obstruction between neighboring RTGs. In the same period, A. Mowery [14] performed statistical analyses of the results of various tests on thermoelectric converters that were similar to the converters in the GPHS-RTGs, but which were electrically heated and which operated at constant thermal powers and constant temperatures. These statistical analyses showed that the measured power output of a constant-temperature converter is a linear function of the square root of time $t$, and that the coefficient of $\sqrt{t}$ is an Arrhenius function of the absolute hot-junction temperature T. In the range of practical interest, Mowery found that the measured effect of thermoelectric degradation could be quite accurately correlated by a simple empirical equation equivalent to

$$
\begin{aligned}
& P / P_{\mu}=1 \cdot\left(\exp \left[-T^{*} / T\right]\left(t / t^{*}\right)\right\}^{1 / 2}, \\
& \text { where } T^{*}=30,960^{\circ} \mathrm{K} \text { and } t^{*}=0.648 \mathrm{sec}
\end{aligned}
$$

This empirical expression was found to give excellent agreement with the experimental data from the electrically heated tests at various constant thermal powers and temperatures. However, Mowery's equations cannot be directly applied to predict the performance degradation of RTGs, because an RTG operates at a diminishing thermal power and temperature, and therefore at diminishing degradation rates. To account for that effect, the present author proposed the following simple modification of Eq. 20.

$$
P / P_{M}=1 \cdot\left\{\int_{0}^{t} \exp \left[-T^{*} / T(t)\right] d t^{\prime} / t^{*}\right\}^{I / 2}
$$

where $\mathbf{T}\left(\mathrm{t}^{\prime}\right)$ is the hot-junction temperature at time $t^{\prime}$. Note that for the special case of constant $T$, the modified equation reduces to Mowery's validated Eq. 20. For variable T, the validity of Eq. 21 was subsequently confirmed by successfully predicting the results of long-term tests at diminishing temperatures.

As shown in Figure 8, the RTG's average hotjunction temperature $T$ is a linear function of the RTG's thermal power $\mathbf{Q}$ :

$$
T=T_{o}+\alpha Q \text {. }
$$

where the coefficients $T_{0}$ and $\alpha$ are obtained from detalled thermal and electrical analysis of the RTGs for various values of $Q$. For example, least-square analysis of the results for two mutually obstructing RTGs separated by an angle of 35 degrees yields the curve-fit coefficients

$$
T_{\circ}=582.6^{\circ} \mathrm{K} \text { and } \alpha=0.1565^{\circ} \mathrm{K} / \mathrm{w} \text {. }
$$

Inserting Eqs. 22 and 17 into 20, we obtain

$\frac{P}{P_{u}}=1-\left\{\int_{0}^{t} \exp \left[-\frac{T^{*}}{T_{0}+\alpha Q_{1} \exp (-\lambda, j)}\right] d i / t^{*}\right\}^{1 / 2}$ 
Defining a dimensionless variable

$$
x \in T^{*} /\left[T_{0}+\alpha Q_{1} \exp (-\lambda i)\right] .
$$

Equation 23 reduces to

$$
\begin{aligned}
& \frac{P}{P_{u}}=1 \cdot\left\{\frac{1}{\lambda i^{*}} \int_{x_{j}}^{x_{2}} \frac{\exp (-x) d x}{x\left[1 \cdot\left(T_{0} / T^{*}\right) x\right]}\right\}^{1 / 2}, \\
& \text { where } x_{i}=T^{*} /\left(T_{0}+\alpha Q_{i}\right) \\
& \text { and } x_{2}=T^{*} /\left(T_{0}+\alpha Q_{1} e^{\left.-\lambda t_{1}\right)}\right.
\end{aligned}
$$

Equation 25 can be partitioned into

$\frac{P}{P_{u}}=1 \cdot\left\{\frac{1}{\left(\lambda i^{*}\right)}\left[\int_{x_{j}}^{x_{2}} \frac{e^{* x} d x}{x}+\int_{x_{1}}^{x_{1}} \frac{e^{-x} d x}{\left(T^{*} / T_{0}\right)-x}\right]\right\}^{1 / 2}$.

for which exact solutions in terms of exponential integrals are readily obtained. However, when we consider the magnitude of the $\mathrm{x}$-values of interest, an almost exact but much simpler solution is available. Let us consider a typical problem with a BOM thermal power $Q_{1}$ of 4410 watts and a mission life $t$ of 12 years. Thus, from Eqs. $25 \mathrm{a}$ and $25 \mathrm{~b}$,

$$
\begin{aligned}
& x_{1}=24.325, \\
& x_{2}=25.590 .
\end{aligned}
$$

For that range of $x$-values, the denominator in the integrand of Eq. 25 varies only from 13.18 to 13.26 . Therefore, using a constant average value for that denominator is accurate to within $\pm 0.3 \%$, and Eq. 25 can be quite accurately approximated by

$\frac{P}{P_{u}} \equiv 1-\left\{\frac{1}{\left(\lambda s^{*}\right) \bar{x}\left[1-\left(T_{0} / T^{*}\right) \bar{x}\right]} \int_{x_{1}}^{x_{3}} e^{-x} d x\right\}^{1 / 2}$

where $\bar{x} \equiv T^{*} /\left(T_{0}+\alpha Q_{1} e^{-\lambda t / 2}\right)$.

Integration of Equation 27 gives

$$
\frac{P}{P_{u}}=1 \cdot\left\{\frac{\exp \left(-x_{j}\right) \cdot \exp \left(-x_{2}\right)}{\left(\lambda t^{*}\right) \bar{x}\left[1-\left(T_{0} / T^{*}\right) \bar{x}\right]}\right\}^{1 / 2},
$$

where $x_{1}, x_{2}$, and $x$ are given by Eqs $25 a, 25 b$, and 27 a. The above solution accounts for diminishing temperatures and degradation rates with time. Finally, Eqs. 19 and 28 can be inserted into 15 to obtain the combined effect of fuel decay and thermoelectric degradation for a given BOM thermal power $Q_{1}$ and mission time $t$.

$$
P=\left(P^{*}+\eta^{*} Q_{I} e^{-\lambda \cdot t}\right)\left\{1-\left[\frac{\exp \left(-T^{*} / T_{1}\right)-\exp \left(-T^{*} / T\right)}{\left(\lambda i^{*}\right)\left(T^{*} / \bar{T}\right)[1-(T d \bar{T})]}\right]^{1 / 2}\right\}
$$

$$
\begin{aligned}
& \text { where } T_{l}=T_{o}+\alpha Q_{t} \\
& T=T_{e}+\alpha Q_{I} e^{-\lambda t} \\
& \bar{T}=T_{o}+\alpha Q_{1} e^{-\lambda t / 2}
\end{aligned}
$$

\section{VALIDATION OF DEGRADATION MODEL}

The above equations were applied to the Q-1 RTG, which was the qualification unit for the GPHS-RTGs used on the Galileo mission. The computed results for a 12-year period are shown in Figure 10. The curve labeled $Q / Q_{1}$ shows the thermal power decrease due to isotope decay (Eq. 17), and the curve labeled T shows the resultant $65^{\circ} \mathrm{C}$ decrease in average hotjunction temperature (Eq. 29b). The curve labeled $P_{u} / P_{1}$ shows the power loss due to fuel decay for undegraded thermoelectric performance (Eq. 19), and the curve labeled $\mathrm{P} / \mathrm{P}_{4}$ shows the power loss due to thermoelectric degradation (Eq. 28). The combined effect of fuel decay and thermoelectric degradation is shown by the curve labeled $P / P_{1}$ (Eq. 15). As can be seen, during the first 3.5 years the thermoelectric degradation effect predominates, and after that the isotope decay effect dominates. The combined effect for that case is predicted to result in a $23.6 \%$ power loss in 12 years.

Figure 10. Effect of Fuel Decay and Thermoelectric Degradation on Performance of Galileo Qualification RTG (Q-1)

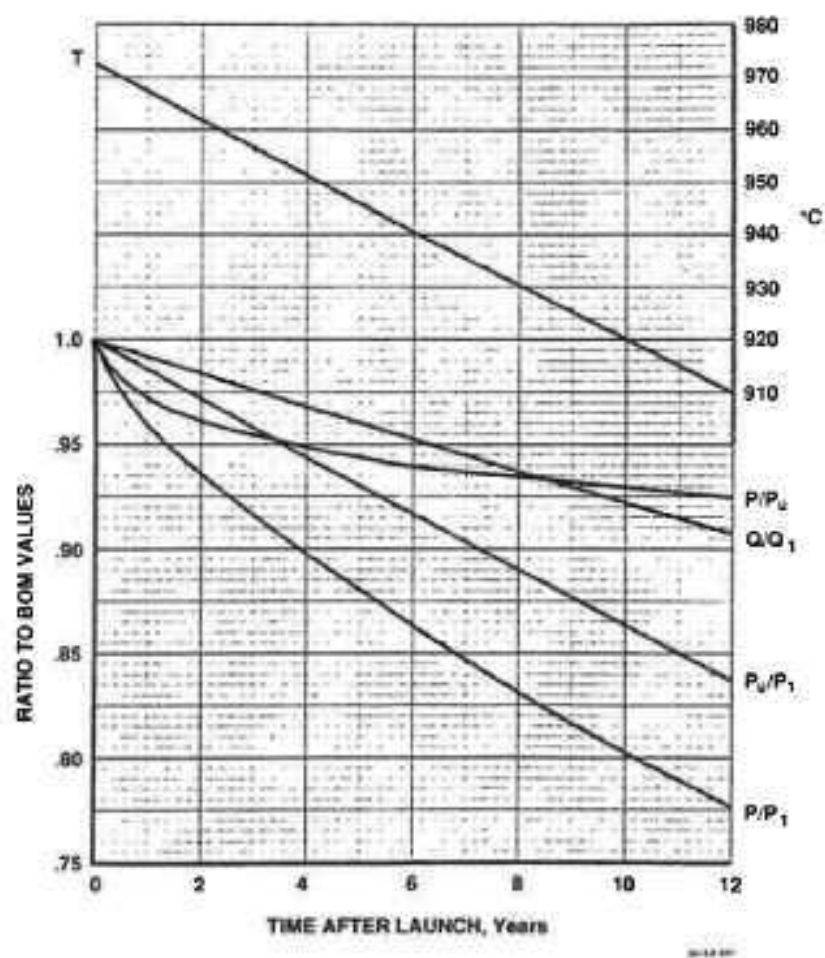

Figure 11 shows a comparison of these predictions with the measured results of a five-year test of the Q-1 RTG at diminishing temperatures. As can be seen, for the range of times and temperatures tested, the rather simple prediction model used showed surprisingly good agreement with the experimental results. 
Figure 11. Comparison of Predicted and Measured Power History of Q-1 RTG

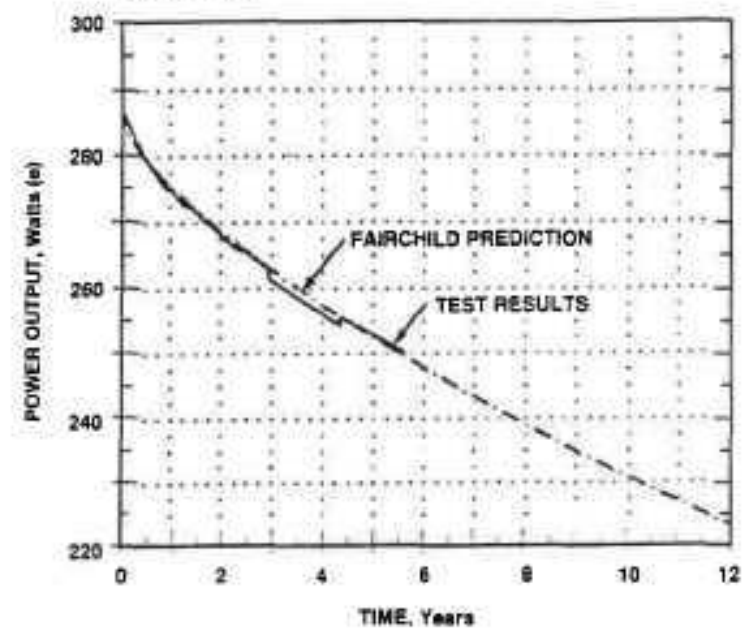

PARAMETRIC PERFORMANCE PREDICTIONS FOR CRAF AND CASSINII FIG

The analytical methodology described above was used to predict the long-term power degradation of the two CRAF and Cassini RTGs integrated with their respective spacecraft. (See Figure 1.) Parametric analytical results for two RTGs with a 35 degree separation angie are shown in Figure 12. For various fuel loadings, the solid curves show the variation of RTG power with time. For each solid curve, the figure shows the thermal power (in each of the 18 heat source modules) and the corresponding plutonium enrichment (for the same fuel density as that used in the Galileo RTGs). The numbers listed are for two points in time: at the chemical separation of the plutonium from the irradiated neptunium-237 targets (BOL), and three years later at launch (BOM). Thus, the figure can be employed as a useful design tool by the CRAF and Cassini mission planners.

For each solid curve in Figure 12, its intersections with the dashed curves indicate the decrease of the RTG's average hot-junction temperature with time for that fuel loading. (Each constant-temperature curve was obtained by inserting $T$ into Eq. 29b, solving for $Q_{1}$ at each mission time, and using that value of $Q_{1}$ in Eqs. 29, 29a, and $29 \mathrm{c}$ to compute the corresponding output power $\mathbf{P}$.)

The bold solid curve in the figure represents the fuel loading in the Galileo RTGs, and the bold dashed curve represents the $1000^{\circ} \mathrm{C}$ hot-junction limit adopted in previous missions. Note that this limit is exceeded by the BOM temperatures at higher fuel loadings. But modest excesses may be allowed, since the results displayed in Figure 12 already include proper allowances for increased degradation at the higher temperatures, based on results of (accelerated) tests conducted above $1000^{\circ} \mathrm{C}$.
Figure 12. Effect of Fuei Loading on RTGs' Power and Temperature Histories $135^{\circ}$ Separation, 10 Heat Source Modules, 36 Couple Rings, $3^{\prime \prime}$ Fins, 30 Volts/RTG]

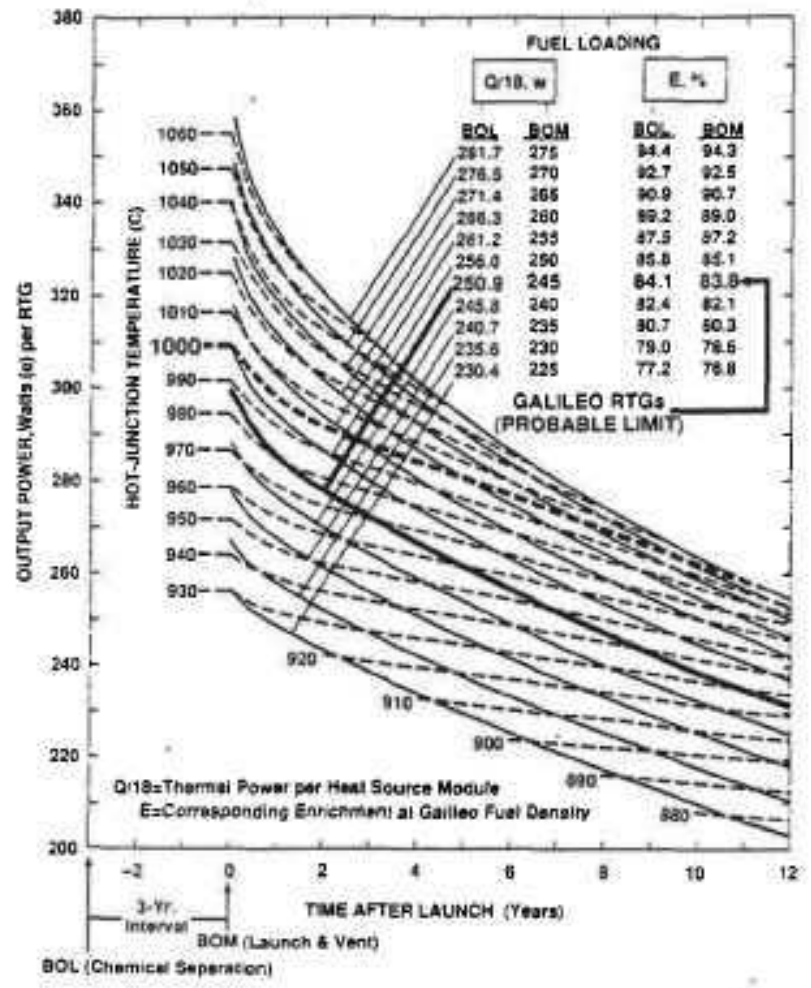

Figure 12 shows that increasing the BOM thermal power (by increasing the fuel loading) leads to a substantial increase of the RTG's electrical power at the beginning of mission, but that this benefit diminishes towards the end of mission, particularly for high fuel loadings and long mission times. This comes about because higher thermal powers result in higher temperatures and therefore higher degradation rates. In fact, at unrealistically high hot-junction temperatures increasing the thermal power can actually decrease the EOM electrical power.

\section{APPUCATION TO TWO UNEQUAL RTGS}

JPL's original CRAF and Cassini mission pians called for the use of two GPHS RTGs on each spacecratt. Under the corresponding DOE plan, two freshly fueled RTGs (F6 and F7) were to be used for the Cassini mission, which has the higher power demand. In that case, Figure 12 could be applied directly to predict the RTGs' power output. However, the CRAF mission under that plan was slated to use only one freshly fueled RTG (F2) and one aged spare unit (F5) left over from the Galileo program. The fuel of that unit was encapsulated in 1982, and will have decayed for over 13 years by the time of the CRAF spacecraft's planned 1996 launch. As a result, by the beginning of the mission its thermal power will have dropped from 252 watts to 227 watts per heat source module. 
During almost all of the 13-year storage interval, the old RTG will have been filled with an argon cover gas, to spoil its multifoil thermal insulation, lower its hot-junction temperature, and virtually eliminate its thermoelectric degradation. Thus, the spacecraft will be launched with two ATGs having similar thermoelectric properties but substantially different thermal powers and temperatures. To determine the effect of the two unequal RTGs on each other, a detalied BOM thermal and electrical analysis of the baseline-configured RTGs with respective thermal powers of 245 and 227 watts per heat source module was carried out. The results fell right on the curves shown in Figures 7 and 8 for obstructed but equal RTGs. In other words, each RTG is affected by the obstruction of its neighbor, but its resultant power loss is essentially independent of modest deviations in its neighbor's thermal power. Therefore, Figure 12 can also be used to predict the performance history of two unequal RTGs by using an appropriate BOM thermal power for each RTG.

\section{PROGRAMMATIC IMPLICATIONS FOR CRAF AND CASSINI MISSIONS}

Prior to September 1990 , only fragmentary information about the power demand for the two missions had been issued by JPL. Preliminary analyses using the above-described Fairchild methodology indicated that JPL's baseline plan would comfortably meet the then-specified power demand for the CRAF mission, and would come close to meeting it for the Cassini mission. The then-existing status is summarized in Table 6.

Table 6. Power Margins in CRAFICASSINI RTGs (Aug. 1990)

\begin{tabular}{|c|c|c|c|c|}
\hline Massion & CRA & & CAS & ssive \\
\hline $\begin{array}{l}\text { Event } \\
\text { Years After Launch } \\
\text { Missien Powor Recuivement (eats) }\end{array}$ & $\begin{array}{c}\text { Rendervous } \\
5.01 \\
450\end{array}$ & $\begin{array}{c}\text { Panhelon } \\
7.61 \\
432\end{array}$ & $\begin{array}{l}\text { Anival } \\
6.50 \\
524\end{array}$ & $\begin{array}{l}\text { EOM } \\
10.30 \\
478\end{array}$ \\
\hline $\begin{array}{l}\text { RTG } \\
\text { ADe of Fual at Lasunch (years) }\end{array}$ & $\begin{array}{ll}1 & 2 \\
3 & 13\end{array}$ & $\begin{array}{ll}1 & 2 \\
3 & 13\end{array}$ & $\begin{array}{ll}1 & 2 \\
3 & 3\end{array}$ & $\begin{array}{ll}1 & 2 \\
3 & 3\end{array}$ \\
\hline S. Modulo (watts) & $\begin{array}{ll}252 & 252 \\
245 & 227\end{array}$ & $\begin{array}{ll}252 & 252 \\
245 & 227\end{array}$ & $\begin{array}{ll}252 & 252 \\
245 & 245\end{array}$ & $\begin{array}{ll}252 & 252 \\
345 & 245\end{array}$ \\
\hline $\begin{array}{l}\text { Hoiluncion Temperature ("C) } \\
\text { At Launch } \\
\text { At Event }\end{array}$ & $\begin{array}{cc}1090 & 952 \\
973 & 924\end{array}$ & $\begin{array}{l}1000962 \\
950910\end{array}$ & $\left|\begin{array}{cc}1000 & 1000 \\
956 & 066\end{array}\right|$ & $\begin{array}{ll}1000 & 1000 \\
946 & 946\end{array}$ \\
\hline $\begin{array}{l}\text { Eloctrical Power (watts) } \\
\text { As Launch } \\
\text { At Event } \\
\text { Tocal at Evert }\end{array}$ & $\begin{array}{c}305 \\
2657 \\
502\end{array}$ & $\begin{array}{c}305257 \\
253226 \\
479\end{array}$ & $\left|\begin{array}{cc}305 & 305 \\
258 & 259 \\
516\end{array}\right|$ & 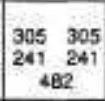 \\
\hline Power Margin (waths) & 44 & 47 & -8 & 4 \\
\hline
\end{tabular}

In late September 1990, as the result of ongoing mission studies, JPL issued much more detailed (and significantly higher) power demand goals to meet the missions' engineering and science requirements. Figures 13 and 14 display these power demand goals for the CRAF and Cassini missions in the form of histograms that have been superimposed on the power supply capability curves for two RTGs. As can be seen, for achievable thermal powers (e.g., 245 watts BOM per module), the original JPL plan talls significantly short of meeting the revised power demand goals, particularly in the case of the Cassini mission.

Figure 13. Power Demand Versus Supply, for CRAF Mission (35* Separation, 18 Heat Source Hodules, 36 Couple Aings, $3^{*}$ Fins, 30 Volts/RTO)

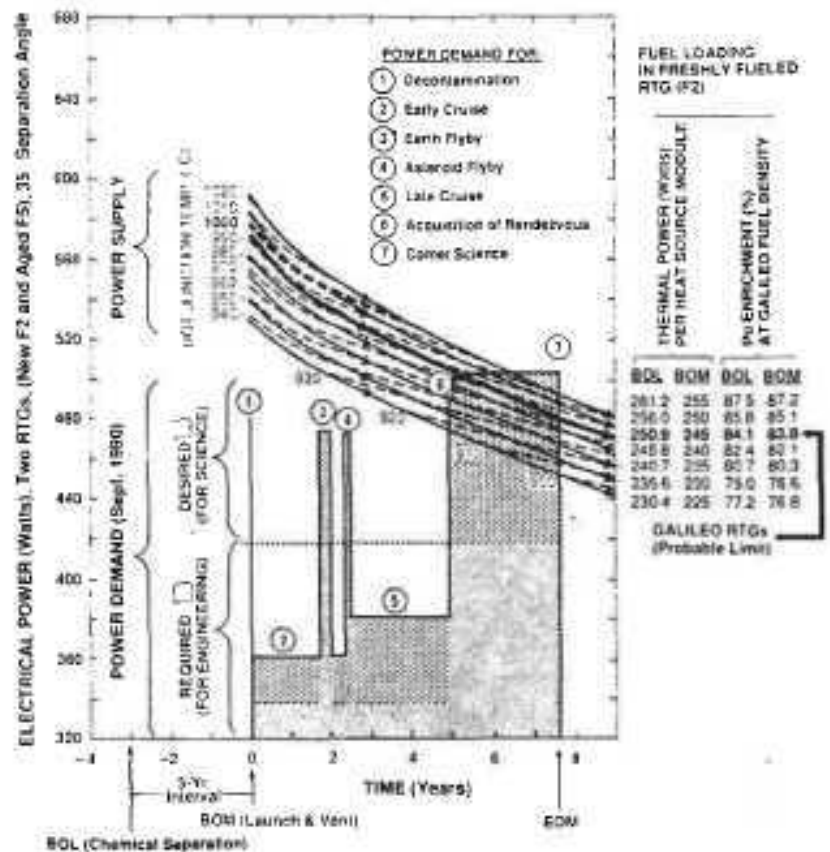

Figure 14. Power Demand Versus Supply, for CASSINI Mission (35" Separation, is Heat Source Modiles, 36 Couple Rings, 3" Fins, 30 Volta/RTO)

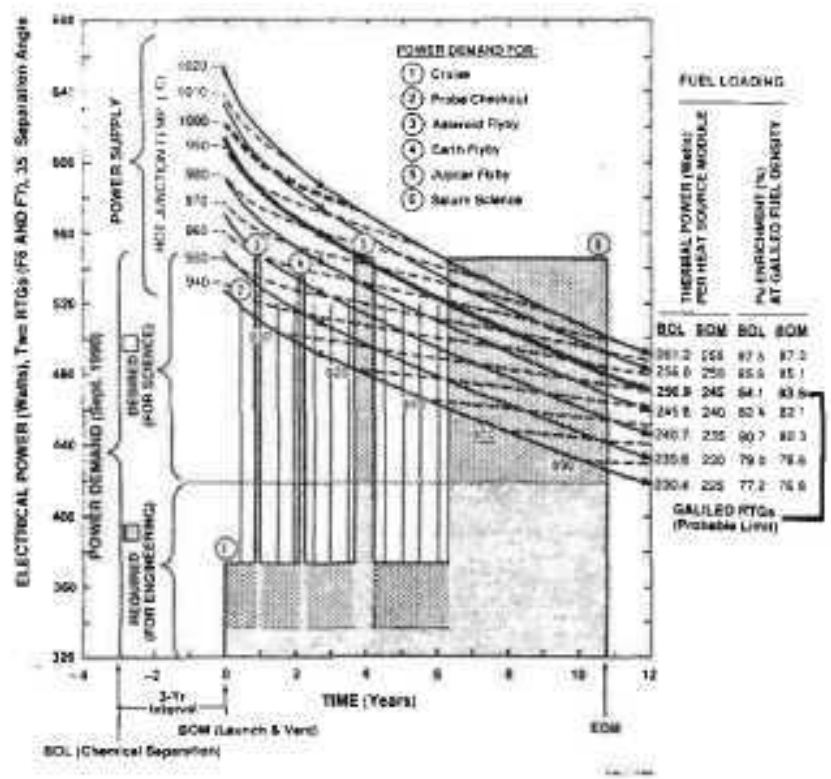


Subsequently, in late October, the power shortiall was further exacerbated when JPL specified additional increases in power demand and mission duration, for both missions. The mission duration for CRAF was increased from 7.6 to 9.4 years, and the duration of Cassini was increased from 10.7 to 12.0 years. These time increases were introduced to allow additional gravity-assist maneuvers, in order increase the spacecraft's mass margins.

Because the twice-revised power demand goals for both missions significantly exceed the capability of two standard RTGs. JPL proposed the use of three instead of two RTGs for each mission. In the case of the Cassini mission, the revised power demand is so high that use of the third RTG appears to be unavoidabie. But in the case of the CRAF mission, there was at least a possibility of meeting the revised power demand profile with two RTGs, by relatively modest modifications of their design and/or operating procedure. If that were possible, it would result in significant cost savings and improved schedule margins. These potential benefits prompted Fairchild to investigate a variety of stratagems, to determine whether they could make it possible to meet the revised power demand for the CRAF mission with just two RTGs.

The magnitude of the problem is illustrated in Figure 15, which compares the predicted power output of two standard GPHS RTGs (for various fuel loadings) with the CRAF power demand goals furnished by R. Campbell, JPL on 11/6/90.

Figure 15. Revised CAAF Power Demand (11-6-90) Versus Output of Two Standard ATGs

(35 Separation, 18 Hest Scurce Modules, 36 Couple Rings, $3^{\circ}$ Fina, 30 Volts/RTG)

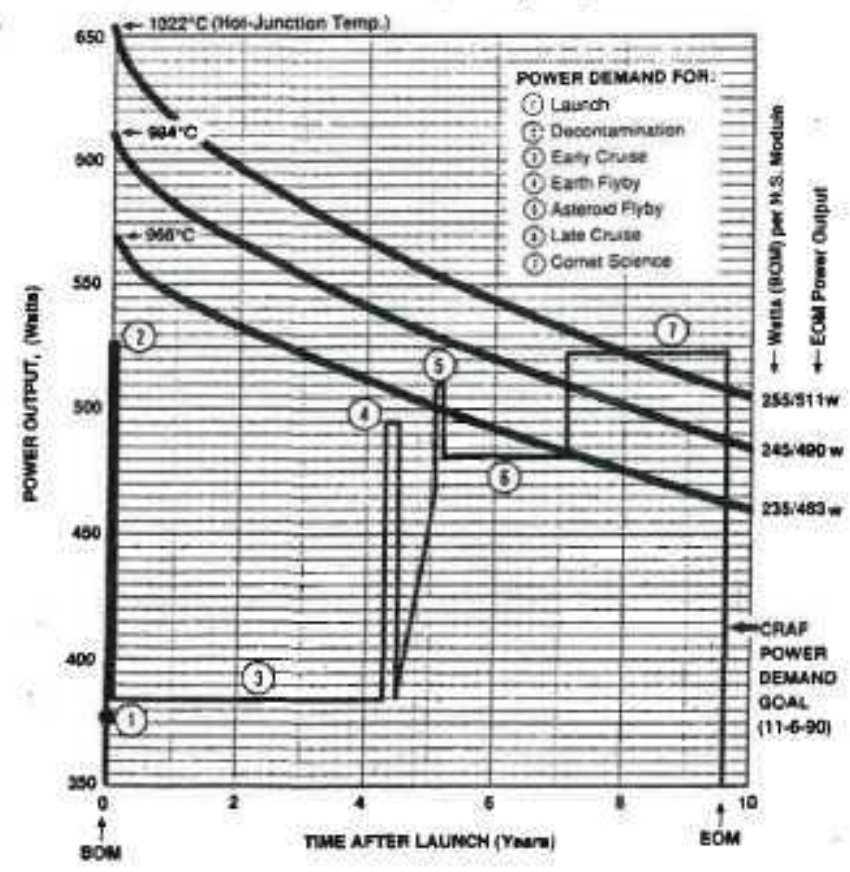

The CRAF power demand profile is shown in the form of a histogram which covers the cruise phase $10<$ $t<7.1 \mathrm{yrs})$ and the comet science phase $(7.1<\mathrm{t}<$ $9.42 \mathrm{yrs}$ ). As can be seen, the two standard RTGs can meet cruise power goals, but with an achievable fuel loading ( 240 to 245 thermal watts BOM per heat source module) they fall short of meeting the JPL. specified 524-watt goal for the comet science phase. For 245-watt heat source modules, the shortfall is seen to be about 13 watts at the beginning and 35 watts at the end of that phase. Thus, unless JPL can reduce its power requirements by that amount (e.g.. by reducing the system-level and subsystem-level contingency reserves built into their estimates), the two RTGs would have to be modified to increase their power output at EOM while still meeting the cruise power demand.

\section{OPTIONS FOR INCREASING THE POWER AT ENCOUNTER}

There are several design or operational modifications that could be used singly or in combination to increase the RTG's power output:

1. "Stretch" the standard RTG, to raise the number of heat source modules from 18 to 19 or 20 .

2. Increase the fuel loading by increasing its density and/or its isotopic enrichment.

3. Delay venting the RTG's cover gas, to reduce the thermoelectric degradation during the coast phase.

4. Reduce the number of thermoelectric couples and/or the leg area in each couple (to increase the temperature drop between junctions and to raise the efticiency).

5. Increase the size of the radiator fins, to decrease the RTGs' cold-junction temperatures.

6. Provide external switching to supply the desired 30-volt output with the two RTGs in series during the power-rich cruise phase and in parallel during the power-lean encounter phases. (Operating each RTG at 15 instead of 30 volts increases the currents and the Peltier cooling rates, which lowers the hot-junction temperatures and the thermoelectric degradation rates.)

7. Use controlled-rate venting of the helium generated by alpha decay to spoil the thermal insulation and lower the hot-junction temperatures and power output just enough to match the power demand profile. This would minimize the thermoelectric degradation. 
The above options, used either singly or in various combinations, were subjected to detailed analyses, to assess their effectiveness in closing the gap between power stypoly and dernand, and to determine which - if any - warrant further investigation. The computed results are presented below. In each case, the results are compared with a histogram of the power demand goals furnished by JPL on $11 / 6 / 90$. It was recognized that these goais were likely to change as the result of changed science demand and as the result of reducing the assumed multi-level contingency reserves. But it was useful to employ the same histogram as a common yardstick, to facilitate comparison of the various options.

"Stretching" the RTG length by 2 or 4 inches to allow raising the number of heat source modules from 18 to 19 or 20 would be a straight-forward method of increasing the ATG's power output, as shown in Figure 16. (Note that the curves for 19 and 20 heat source modules were obtained by simple scaling, which implicitly assumes the same efficiency as the 18. module case. This is conservative, since efficiency generally increases with power output, because end losses constine a smaller fraction of the thermal power in largei RTGs.) As can be seen, even the 19. module ATG would virtually meet all of JPL's power demand goals. However, such a design change would constitute a significant deviation from the standard RTG design, and would therefore require some new qualification tests, which could not be accommodated within the prescribed mission schedule.

Figure 16. Efieet of "Stretching" the Standard ATG (35-5eparalion, 245 Watts BOM per Heat Source Module, 36 Couple Fings, 5 Fins, 30 Volts:ATG)

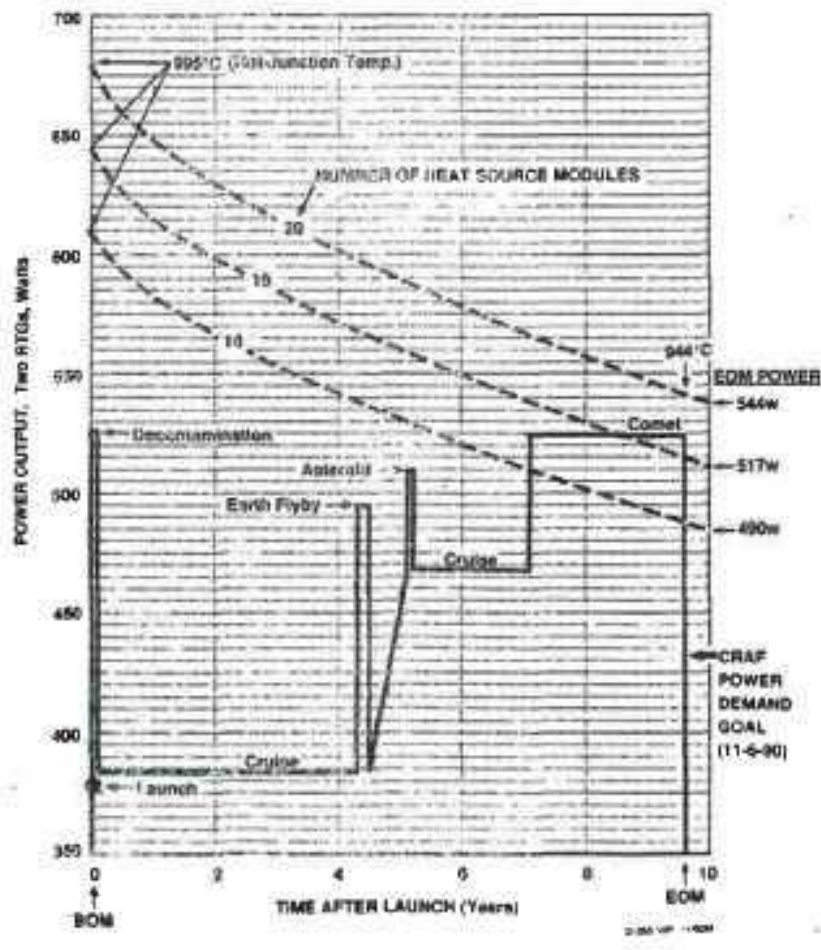

Increasing the fuel loading is another obvious stratagem for raising the RTGs' output, since it would increase the electrical power by raising both the thermal power and the converter efficiency. As shown by Figure 15, increasing the BOM thermal power to -265 watts per heat source module would raise the EOM power to the specified level. But this option may not be doable, at least within the available time.

Significantly increased fuel density, which can be achieved by using higher pressures and/or temperatures during pellet formation, may lead to increased fuel cracking, even before impact; and would probably require additional safety tests, which can be quite costly and time consuming.

Alternatively, increasing the fuel enrichment may not be possible without new fuel production, which probably will not be available in time in the required quantities. Even if higher-enrichment fuel did become avallable, beyond a certain enrichment the resultant higher fuel temperature could also lead to problems in pellet formation requiring costly and time-consuming new fabrication development and safety tests. In any case, until new fuel is available we are limited to the present inventory. A recent study by $M$. Eck [16] indicates that the present inventory, by proper selection, would yield an isotopic enrichment of $82.9 \%$ at the time of chemical separation of the plutonium. This corresponds to a BOL thermal power of 247.3 watts per heat source module, which would decay to a BOM power of 241 watts at launch, 3.25 years later.

The third stratagem for raising the encounter power, delayed venting of the RTG's cover gas, could be used to spoil the converter's multifoll thermal insulation until the mission's comet phase, when the maximum output power is needed. Spolling the thermal insulation would significantly decrease the RTG's hotjunction temperatures and therefore its degradation rate during the mission's cruise phase, making more power available during the comet phase. Unfortunately, the same cover gas that increases the comet science power also diminishes the cruise power. Thus, delayed venting could be quite useful for missions in which the required ratio of cruise power to encounter power is low, but much less useful for a mission like CRAF where that ratio has quite a high value $(468 w / 524 w=89 \%)$.

Figure 17 presents the results of the Fairchild analysis of two RTGs with 245-watt (BOM) heat source modules for three different cover gases: xenon, argon, and a mixture of $73 \%$ argon and $27 \%$ helium. These 
are the only cover gases for which RTG test data are available. (Additional tests to expand the data base are under way at Mound.) This figure assumes venting of the cover gas after eight years, and confirms that delayed venting has the anticipated effect of increasing the power at EOM. The eight-year venting delay would increase the power of the two RTGs at 12 years by about 30 watts for the argon-helium mixture, aimost as much for pure argon, and about 21 watts for the least conductive gas, xenon.

Figure 17. Eflect of Delayed Venting for Various Cover Gases

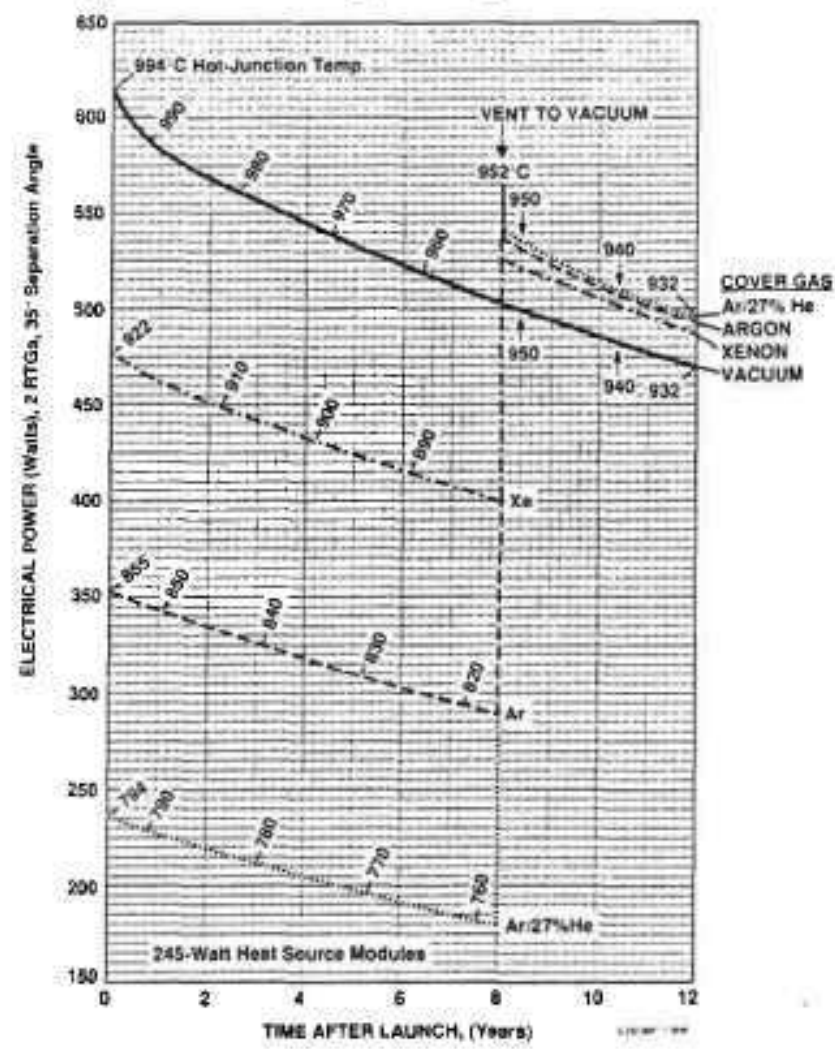

These EOM power increases are respectable improvements, but unfortunately all of these cover gases lead to excessive losses during the mission's cruise phase. The least excessive power loss is obtained with xenon, the inert gas with the lowest thermal conductivity. However, to be effective this would require a means of preferentially discharging the alpha-generated helium while retaining the xenon, e.g. by use of a selective vent. Selective vents made of Viton were used in the Viking RTGs, but the adequacy of such vents for the present application has not been demonstrated.
The effect of delayed xenon venting is illustrated in Figure 18. Two output profiles are shown, one for venting at the beginning of the comet science phase (7.1 yrs), and one for venting just prior to the Earth flyby $(4.2 \mathrm{yrs})$. As can be seen, the power demands for Earth flyby, asteroid flyby, and late cruise cannot be met if venting is delayed until comet rendezvous, but can be met if the RTG is vented just before Earth flyby. Neither option meets the power demand peak for decontamination. Both options meet the demand goal at the beginning of the comet science phase, but neither meets it at the end of that phase. The 7-year vent case misses the required EOM goal by 16 watts, and the 4.2-year vent case misses by 22 watts. Both of these power deficits are smaller than for the vent-atlaunch case ( 35 watts), but they are still not good enough. Does that mean that the use of delayed venting is of no interest? Not necessarily. It may not be adequate when used by itself, but may be adequate in conjunction with one of the other stratagems (e.g., reduced number of couples).

\section{Fgure 18. Effect of Delayed Xenon Vent on Output of Two Standard RTGs (35' Separation, 18 Heat Source Modulies, 35 Couple Rings, 3" Fins, 30 Volts:RTG)}

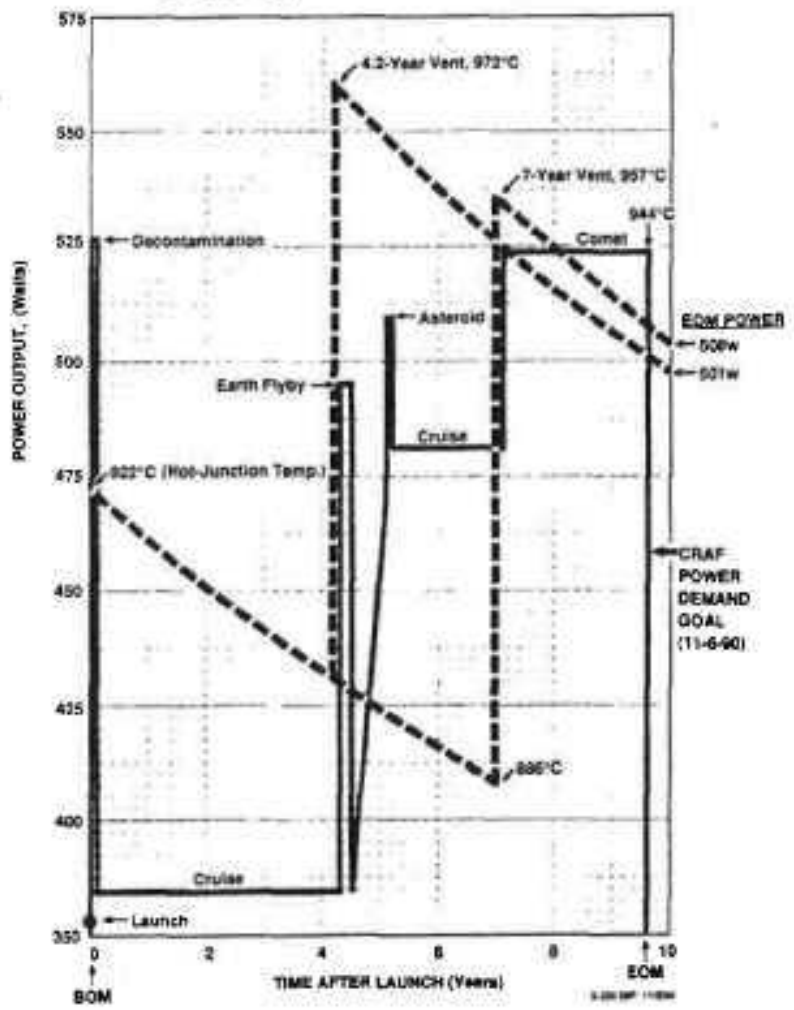

The fourth stratagem involves reduction of the thermoelectric area, either by reducing the number of couples in the RTG, or by reducing the leg area in each couple. The former option is easier to implement, because it avoids the need of developing a new couple. In either case, reducing the thermoelectric 
cross-section of the RTG increases the temperature drops between hot and cold junctions, which increases the conversion efficiency and therefore the RTG's power output for a given thermal power. The deleted couple rings were removed trom the two ends of the RTG. This has the added benefit of improving the uniformity of the hot-junction temperatures, by compensating for the drop-off due to end losses (see Table 3).

Figure 19 compares the performance of the standard (vented-at-launch) 36-ring RTGs with those of the 34-ring and 32-ring designs; i.e., designs in which one or two rings have been deleted from each end of the RTG. As seen, these deletions lead to a substantial increase in the BOM output power. But, as shown, they do so only at the cost of substantially higher hot-junction temperatures. The consequent rise in degradation rates largely defeats the purpose of deleting the couple rings. As can be seen, for the 34 ring case increased degradation reduces the 27-watt gain in BOM power to only 4 watts by the end of mission; and for the 32-ring design the BOM power gain of 53 watts is reduced to a 13-watt power loss at EOM. All three cases fail to meet the EOM power demand goal. Thus, deletion of couple rings is not useful by itself, and would only be useful in conjunction with another method, to reduce the hot-junction temperature. Either delayed venting and/or longer fins may be used for that purpose.

Figure 19. Effect of Deleting Unicouple Rings from Ends of 36.Ring RTGs

(35' Separation, 18 Heal Source Modules, 245-Watt BOM, 36 Couple Rings, 3 " Pins, 30 Volts,ATG)

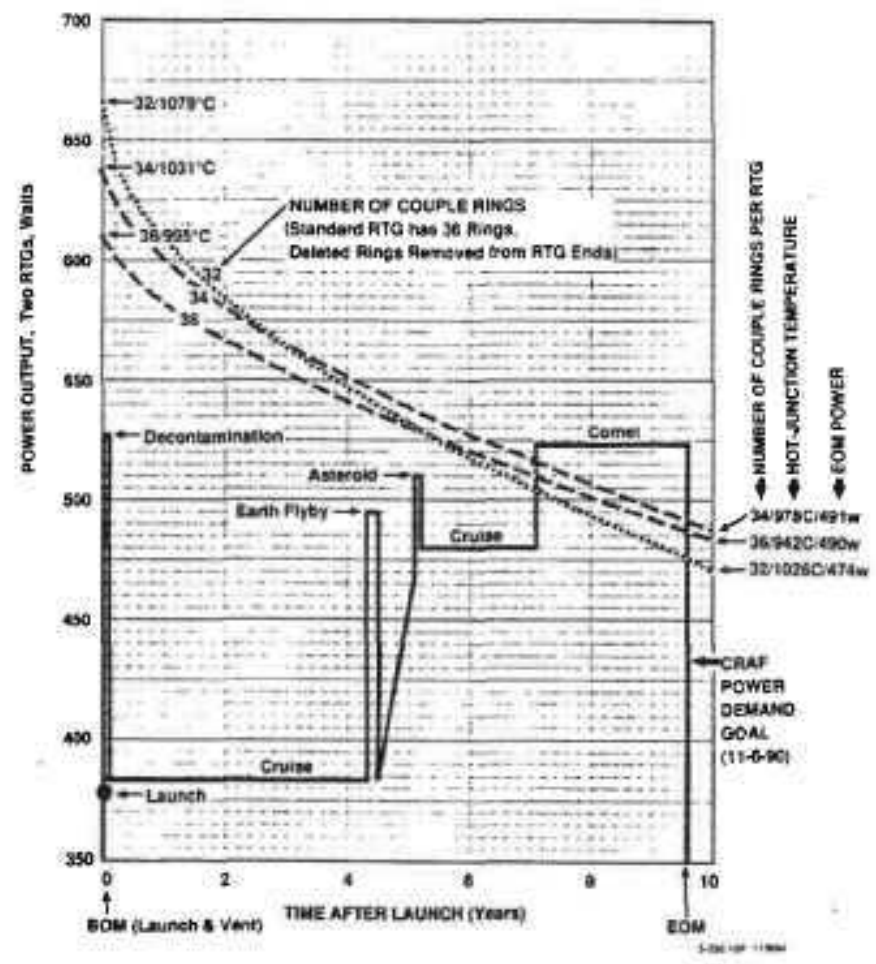

Figure 20 displays the effect of delayed venting on an RTG with 32 couple rings. Results are shown for venting at launch and at 4.2, 5.0, and 7.0 years after launch. As expected, the later the venting the lower the degradation and the higher the EOM power. For venting at 7.0 years, the EOM power is increased by 45 watts, which essentially meets the EOM power demand goal. But as shown, it does not meet the power goal for Earth and asterold flyby or for late cruise. Venting at 5.0 years meets the asteroid and late-cruise goals, but not the Earth-flyby goal. The only strategy that meets the Earth, asteroid, and latecruise goals requires venting at 4.2 years, but this yields an EOM power gain of only 28 watts, which is 22 watts short of the EOM power goal.

\section{Figure 20. Effect of Delayed Xenon Vent on Output of Two} 32-Ring RTGs

(18 Heat Soutce Modules, 245-watt BOM, 3" Fins, 30 Volta:

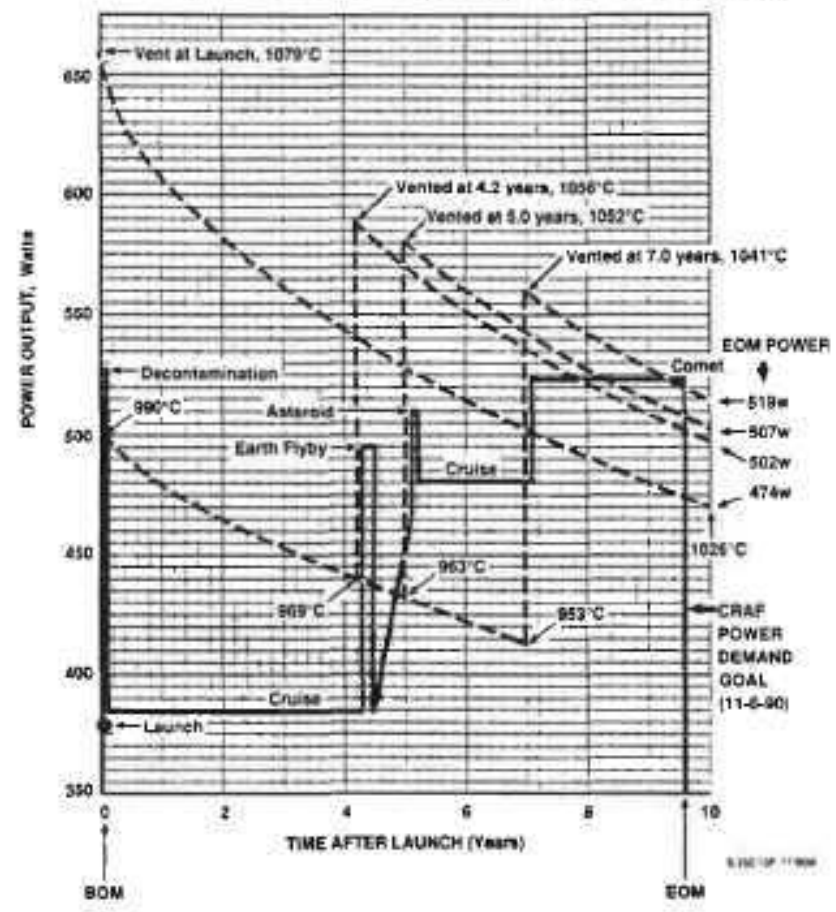

Figure 21 examines the effect of varying the number of couple rings on the output of RTGs vented at 4.2 years. As can be seen, the 34 -ring design results in reasonable temperatures and yields the highest EOM power (508 watts, which is still 16 watts short of the EOM goal).

The effect of increased fin size on performance is depicted in Figure 22. The fins have a trapezoidal cross section with a $0.015^{\text {n }}$ fin tip thickness. The standard design has a $0.060^{\prime \prime}$ fin root thickness with a $3^{\prime \prime}$ root-to-tip height. This is compared with a $5^{\prime \prime}$ fin having a $0.100^{\prime \prime}$ root thickness. The larger fins lower the RTGs's housing temperatures and cold-junction temperatures. But, as shown, they also lower the hot- 
Figure 21. Eflect of Number of Couple Rings on Output on Two RTGs Vented at 4.2 Years (18 Heat Source Modules, 245-Watt BOM, 3"Fins, 30 Voltg/RTG)

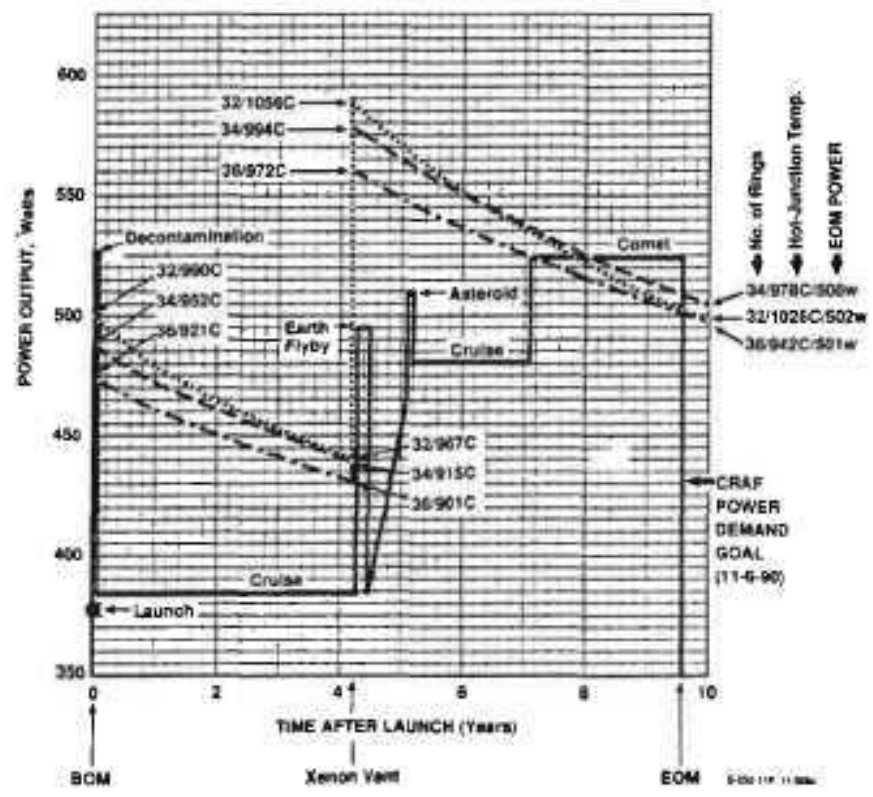

junction temperature by $19^{\circ} \mathrm{C}$, so that there is virtually no change in $\triangle T$ or in BOM power. However, the lower hot-junction temperature does reduce the degradation rate, resulting in a 9-watt increase in EOM power. Thus, while larger fins are helpful, their benefit is not nearly enough to meet the EOM power goal. But it may be a useful design change in conjunction with other modifications.

Figure 22. Eflect of Fin Size on Output of Standard RTGs (35' Separation, 18 Heat Source Modules, 245-Watt BOM, 36 Rings, 30 VolssiRTG)

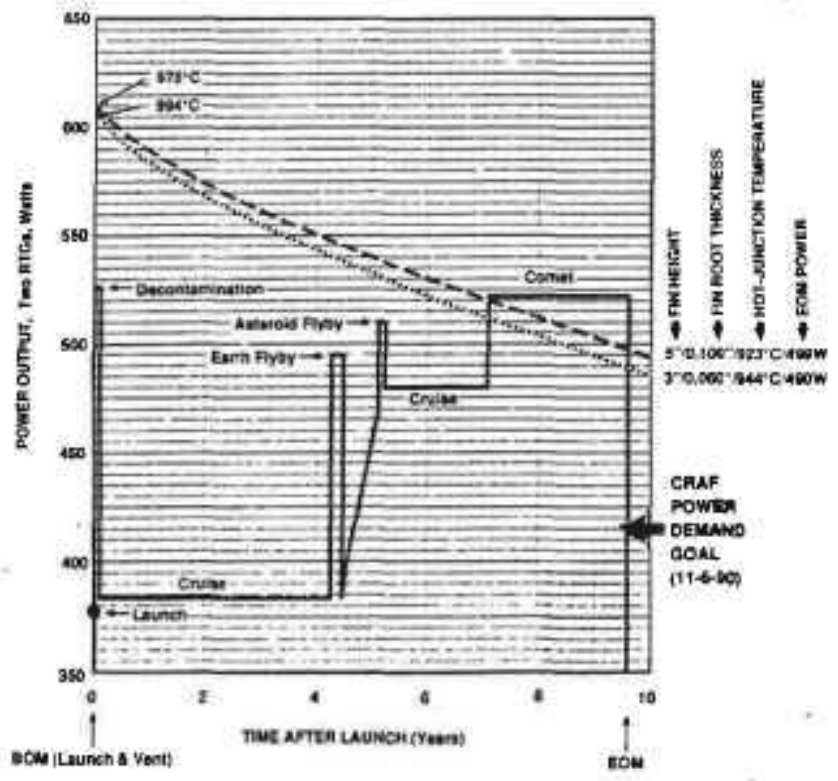

The next stratagem investigated is voltage switching. This is achieved by the addition of an external two-position switch which allows the two RTGs to be connected either in parallel or in series. The alternative circuit configurations are depicted in Figure 23. In either position, the RTGs are connected to a 30 -volt load. As usual, the load voltage is regulated by a shunt dissipator. When the two RTGs are in parallel, each has an output of 30 volts. When they are in series, each has an output of 15 volts.

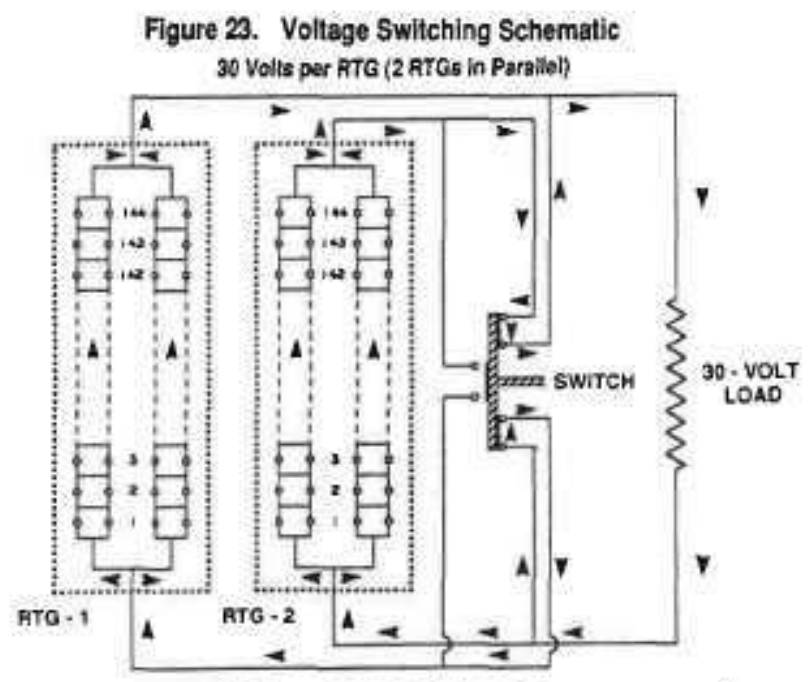

15 Voits per ATG (2 RTGs in Series)

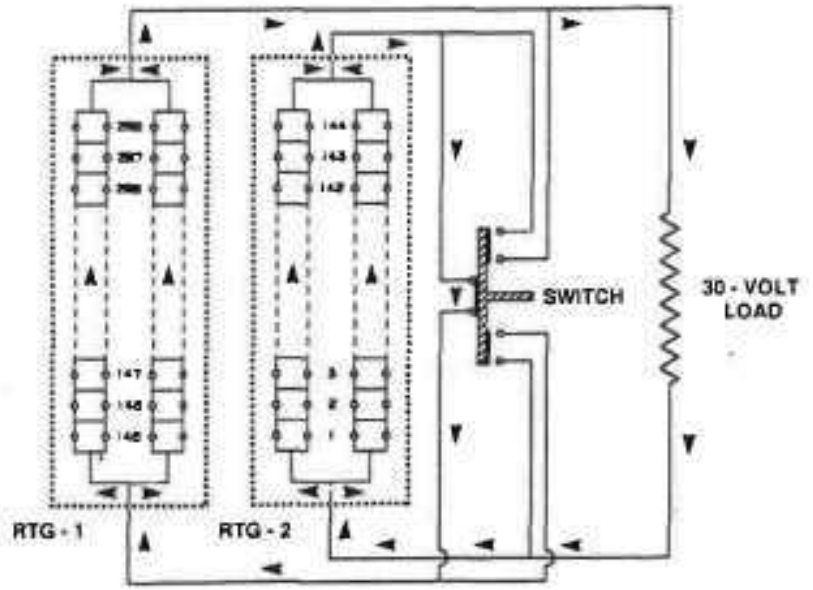

The resultant effects for a 34-ring RTG with 5" fins are shown by the curves of Figure 24, which were obtained by detailed analyses of the obstructed RTG at various voltages. The curve labeled "current" depicts the current-voltage characteristic of each RTG, and the other two curves show the corresponding variations of the output power and the hot-junction temperature. As can be seen, an output of 30 volts is close to the maximum-efficiency point ( 35 volts) while an output of 15 volts is way off-optimum. Lowering the ATG voltage to 15 volt drops the BOM power from 320 watts to 226 watts. But that would be acceptable during mission phases when the power demand is low. 
Figure 24. Effect of RTG Voltage on BOM Power, Current, and HolJunction Temperatures

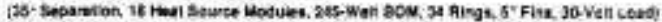

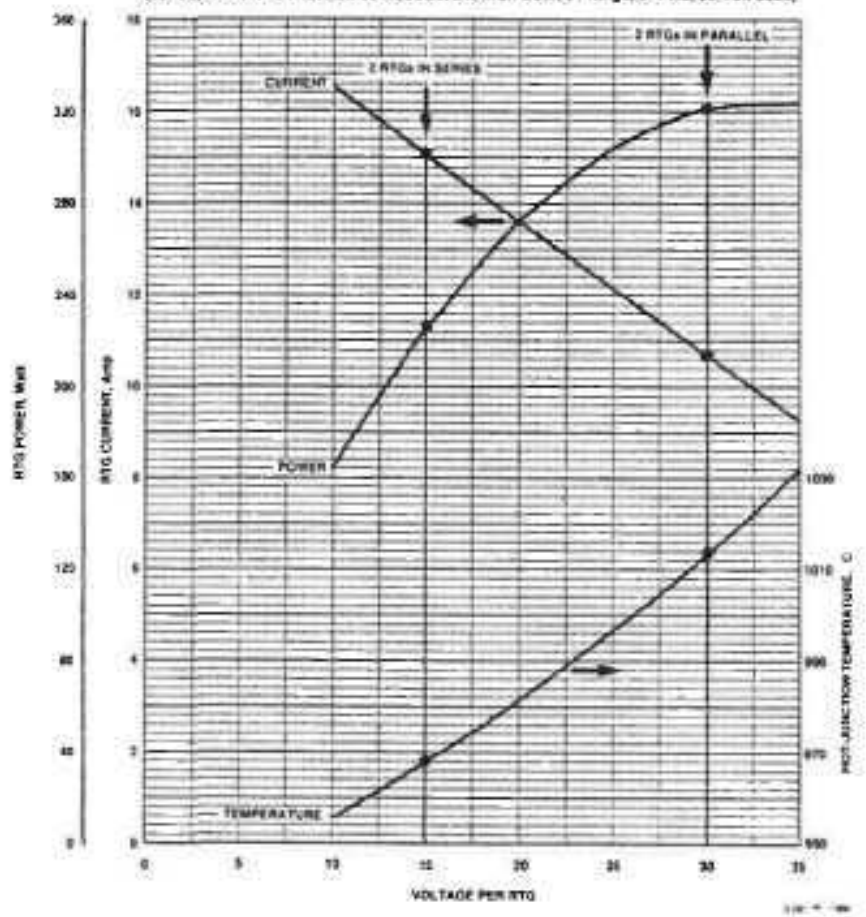

As shown in Figure 24, lowering the RTG's voltage from 30 to 15 volts raises its current from 10.7 to 15.1 amps. This increases the Peltier cooling rate, and as shown - lowers the hot-junction temperature from $1014^{\circ} \mathrm{C}$ to $968^{\circ} \mathrm{C}$. The $46^{\circ} \mathrm{C}$ reduction in hot-junction temperature would greatly decrease the thermoelectric degradation rates during periods when the lower power output is sufficient to meet the demand.

This is illustrated in Figure 25, which shows the effect of voltage switching on the output of a 34-ring

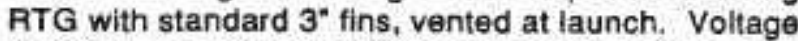
switching has a similar effect as delayed venting. But one of its advantages is that - unlike delayed venting the process is reversible, and can be invoked repeatedly to meet temporary power demand peaks. Another of its advantages is that it does not require a selective vent for preferential release of the helium.

In the case illustrated by the dash-dot curve of Figure 25, the two RTGs operate in series (at 30 volts each) during the brief decontamination cycle at the beginning of the mission, during the Earth flyby, and during the asteroid flyby and the remainder of the mission. During the early cruise phases the two RTGs are in series, at 15 volts each, to reduce the thermoelectric degradation. As can be seen, this strategy meets all of the power demand goals except at the latter part of the comet science phase. Although the 505-watt EOM output for this case is seen to be 14 watts higher than what would be obtained without voltage switching, it is still 19 watts below the EOM power goal.
Figure 25. Effect of Voltage Switching on Output of 34-Ring RTGs Vented at Launch

(35' Separation, 18 Heat Source Modules, 245-Watt BOM, 3" Fins. $15 / 30$ Volts/RTG

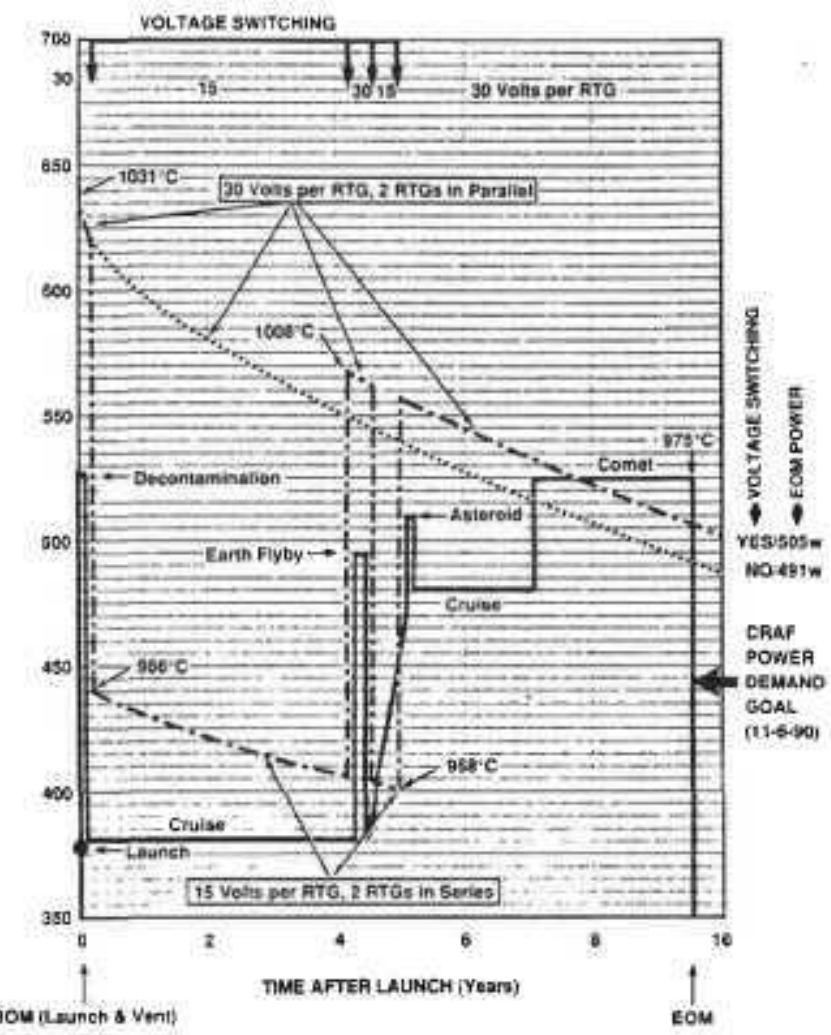

Figure 25. Effect of Fin Size on Output of Dual-Voltage RTGs $\left(35^{\circ}\right.$ Separation, 18 Heat Sourca Modules, 245-Wart BOM, 34 Couple Aings)

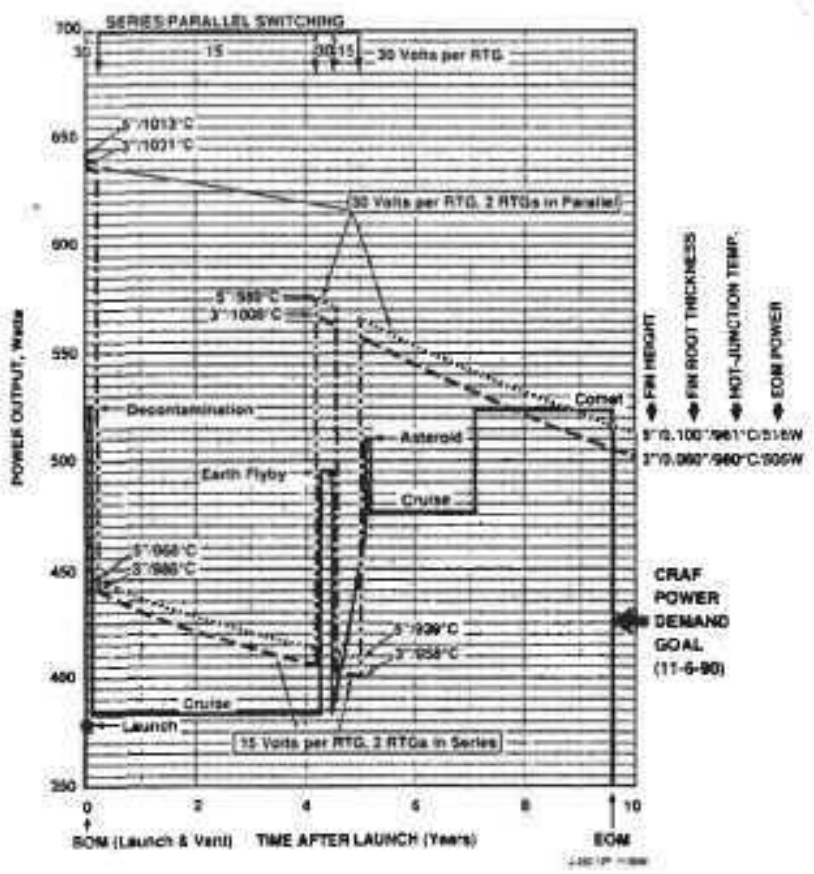


One way of further increasing the RTGs' power output is to combine voltage switching with larger radiator fins. As shown in Figure 26, this increases the EOM power by 11 watts (from 505 to 516 watts), and meets all of the power demand goals except those for the comet science phase. It exceeds that goal by 17 watts at the beginning of that phase, but falis 8 watts short of meeting it at the end of that phase.

Figure 27. Eflect of Number of Couple Rings on Output of Dual-Voltage RTGs

(35० Separation, 18 Hea: Source Modules, 245-Watt BOM, 5 " Fins)

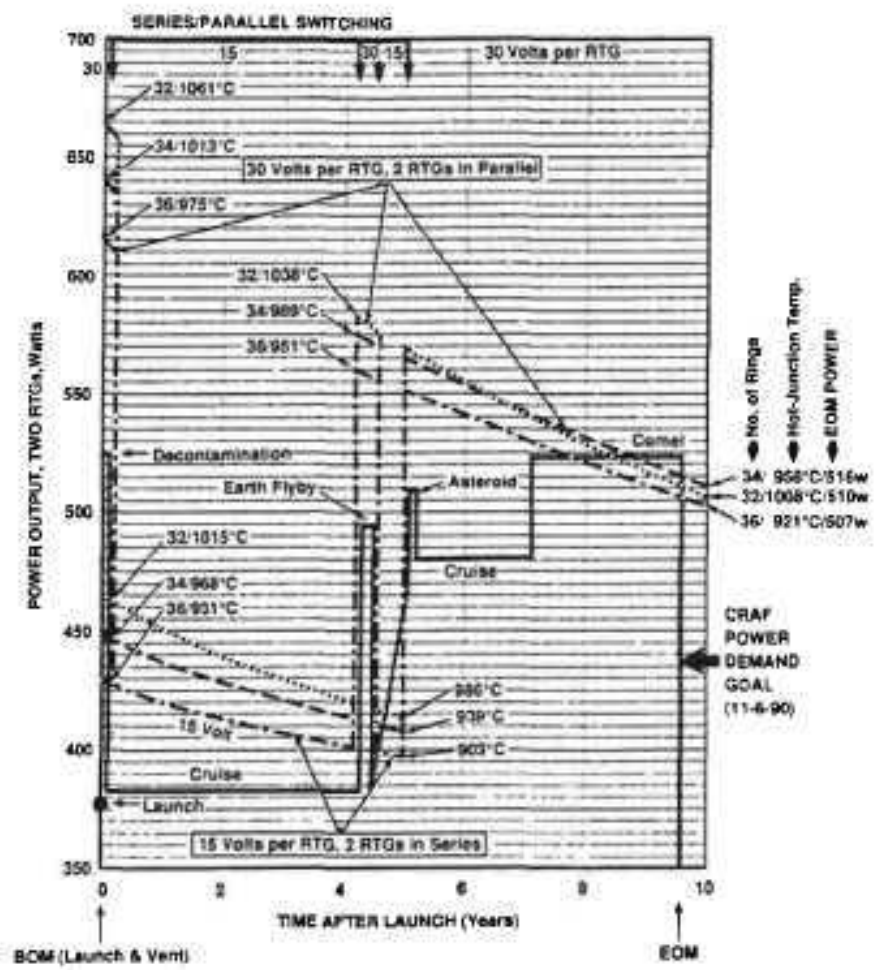

Two more figures are shown to complete the picture for this option. Figure 27 depicts the effect of the number of couple rings on the output of the voltage-switched RTGs with $5^{*}$ fins, and again shows 34 rings to be optimum. Finally. Figure 28 examines the effect of fuel loading on the output of 34-ring RTGs with voltage switching and $3^{\prime \prime}$ fins. It shows that if the BOM thermal power could be raised to 255 watts per heat source module, the EOM power goal could be met without changing the fins from their standard size.

The final stratagem analyzed requires controlledrate venting of the helium generated by the isotope's alpha decay. As indicated earlier in Figure 17, helium is such a good conductor and so effective in spoiling
Figure 28. Effect of Fuel Loading on Output of Dual-Voltage 34-Ring RTGs with 3 " Fins (35" Separation, 18 Heat Sourco Modules, 13/30 Volts por RTG)

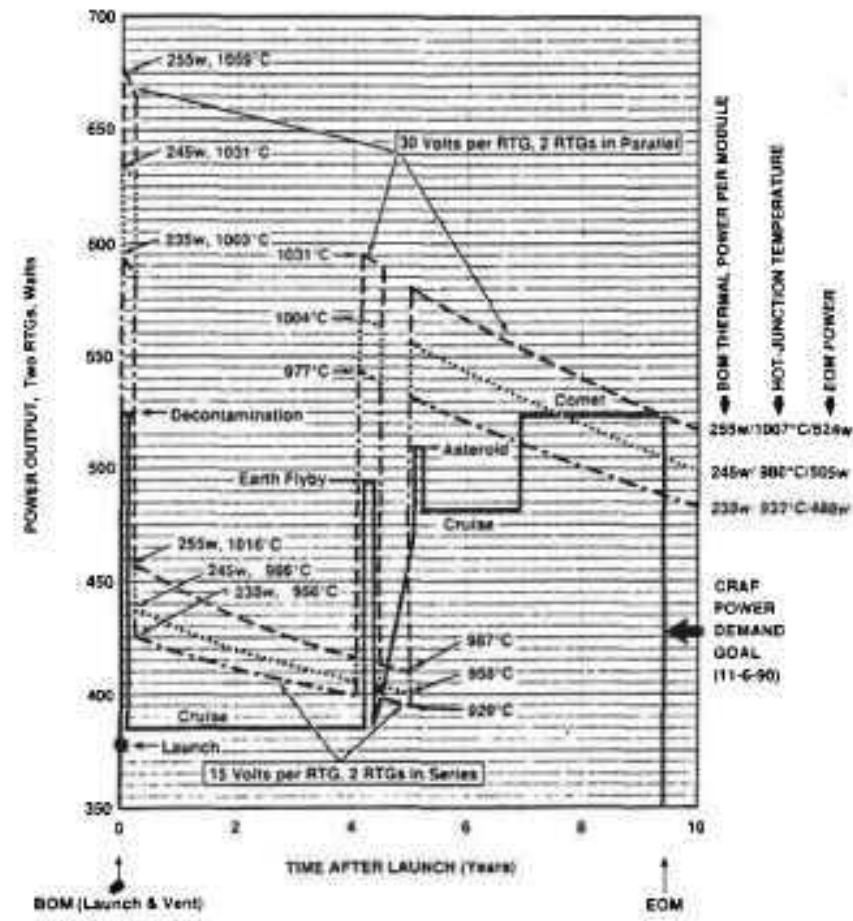

the thermal insulation that its retention above a continuum pressure would reduce the RTG's power output below the level required for the cruise phase. But if the helium pressure were low enough to be in the molecular-flow or transition regime, its thermal conductance could be low enough to yield the required power output. In principle, by careful control of the ATGs' helium pressure their power output could be regulated to just match the power demand histogram. Since that would minimize the hot-junction temperature history and thermoelectric degradation for the prescribed output profile, it would maximize the EOM power output.

The previously described analysis methods for vacuum RTGs must be modified to account for the effect of the lower pressure helium cover gas. Figure 29 shows the experimentally measured effect of helium pressure on a GPHS-RTG's power output $\mathbf{P}_{\mathrm{He}}$ and thermoelectric temperature drop $\Delta T_{H e}$ relative to their respective vacuum values $P_{v}$ and $\Delta T_{V}$. The data plotted rupresent recent measurements by Mound [17] on the Galileo Qualification Unit $(Q-1)$ after a five-year test. The measurements covered a pressure range from vacuum to 20.5 torr. As expected, the fractional decrease in power is approximately twice as great as that in $\Delta T$. 
Figure 29. Effect of Helium Pressure on RTG Power and Temperature

(Measured at Yound on 0-1 RTG)

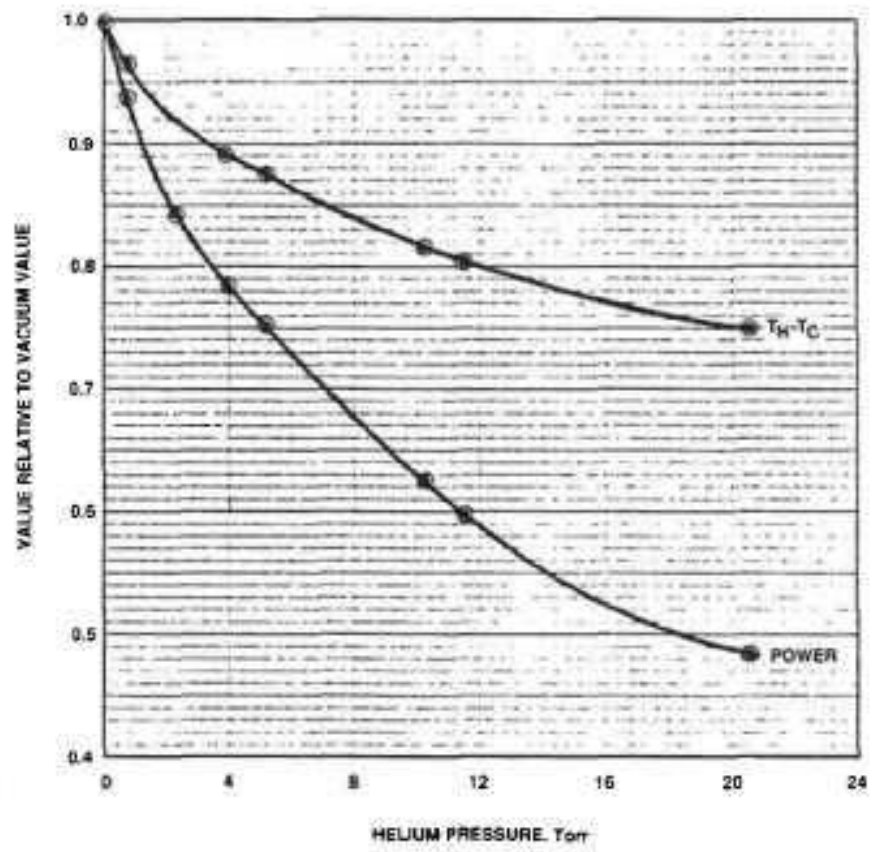

As can be seen, at 20.5 torr the effect of increasing helium pressure on power and $\Delta \mathbf{T}$ is diminishing but has not quite leveled off yet. In other words, helium at that pressure is still somewhat below the continuum regime for this geometry. Extrapolation of the curves suggests that for this RTG geometry helium reaches a continuum at about 24 torr.

Figure 30. Effectiveness of Controlled Helium Pressure in Lowering Temperature at Partial Power

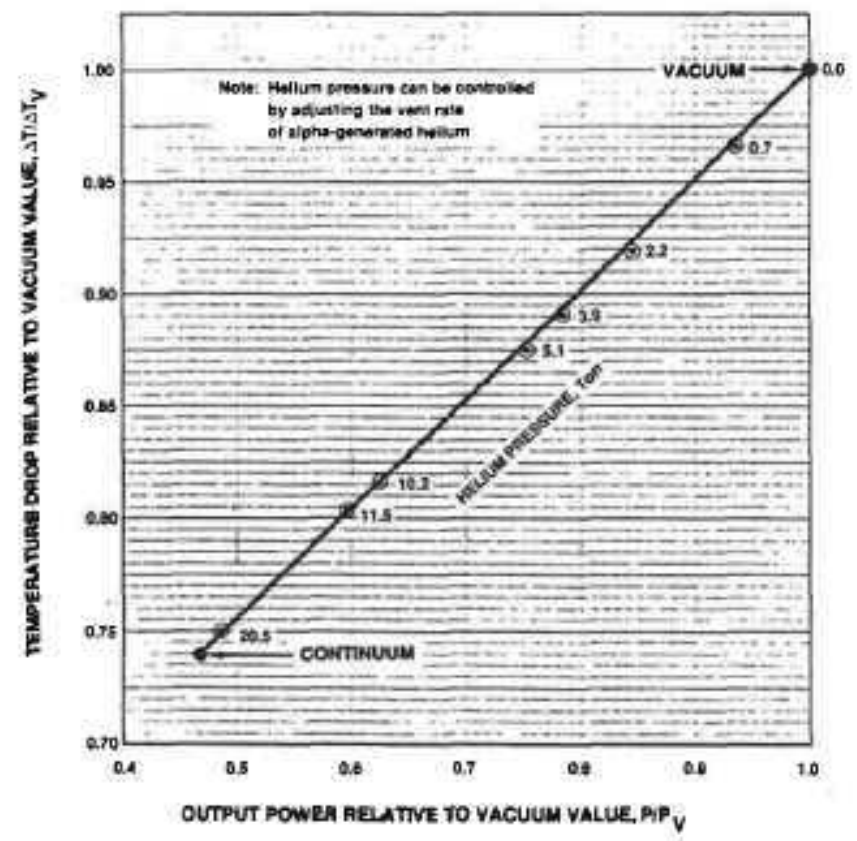

The experimental data are cross-piotted in Figure 30. The plot shows a linear relationship between temperature drop and power output. Note that the lower left terminus of the plot represents the case of a hellum continuum. The plot should not be extrapolated beyond that point, since further increase of the helium pressure does not affect the ATG's temperatures or power output.

Figure 30 can be represented by the simple equation

$$
\frac{T_{H e}-T_{c}}{T_{V}-T_{c}}=I-\beta\left(I-\frac{P_{H e, u}}{P_{V, \mu}}\right) .
$$

where $T_{\mathrm{He}}$ and $\mathrm{T}_{\mathrm{V}}$ are the hot-junction temperatures with and without helium, $\mathbf{T}_{\mathrm{c}}$ is the cold-junction temperature, $P_{H e, u}$ and $P_{V}, u$ are the RTG's undegraded power output with and without helium, and $\beta=0.485$ trom the slope of Figure 30. (In the absence of further experimental data, the analysis assumes that Figure 30 applies not only to the Q-1 RTG but also to any similar SiGe RTG throughout its mission.)

From Eqs. 22 and 17 the hot-junction temperature $T_{V}$ of the vacuum-FTG at time $t$ is given by

$$
T_{V}=T_{e}+\alpha Q_{l} \exp (-\lambda t)
$$

and from Eqs. 16 and 17 its undegraded output power $P_{V, u}$ is given by

$$
P_{V, u}=P^{*}+\eta^{*} Q_{l} \exp (-\lambda t) \text {. }
$$

From Eq. 21, the ratio of degraded power $\mathrm{P}_{\mathrm{He}}$ to undegraded power $\mathbf{P}_{\mathbf{H e}, \mathrm{u}}$ is given by

$$
\begin{aligned}
& P_{\text {He }} / P_{\text {HeN }}=1 \cdot \sqrt{I} \\
& \text { where } I \equiv \int_{0}^{t} \exp \left[-T^{*} / T_{H e}(t)\right] d t t^{*} .
\end{aligned}
$$

Substituting $T_{V}$ from Eq. 31, PV,u from Eq. 32, and

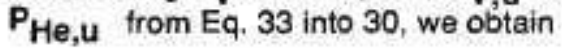

$$
T_{H e}=T_{c}+\left(T_{0}+\alpha Q_{l} e^{-\lambda t}-T_{c}\right)\left[1-\beta\left(1-\frac{P_{H e} I(I-\sqrt{I})}{P^{*}+\eta^{*} Q_{l e}-\lambda t}\right)\right]
$$

for the hot-junction temperature required to match the prescribed power demand $\mathrm{P}_{\mathrm{He}}$ at time $\mathrm{t}$.

At every time step $t$, we obtain $P_{V, u}$ from Eq. 32 and $P_{H e, u}$ from Eq. 33, compute the ratio $\left(P_{\mathrm{He}, \mathrm{u}} / \mathrm{P}_{\mathrm{V}, \mathrm{u}}\right.$ ) and refer to the power curve of Figure 29 to obtain the required hellum pressure at time $t$. We also solve Eq. 33 for the value of the integral I and insert it into Eq. 34 to obtain a new value of $\mathbf{T}_{\mathrm{He}}$ for the next integration step. This cycle is repeated until EOM. 
The results of this procedure are illustrated in Figures 31 and 32 . In both figures, the CRAF power demand profile is shown by the dotted histogram, and the power output of the two RTGs is displayed by the dashed curve. Figure 31 is for two standard RTGs, and Figure 32 is for two RTGs with 32 instead of 36 couple rings.

\section{Figure 31. Eflect of Controlled-Rate Venting of Helium on Output of 36-Ring RTGs}

(35 Separation, 18 Heat Source Modules, 245-Watt BOM, 3" Fins)

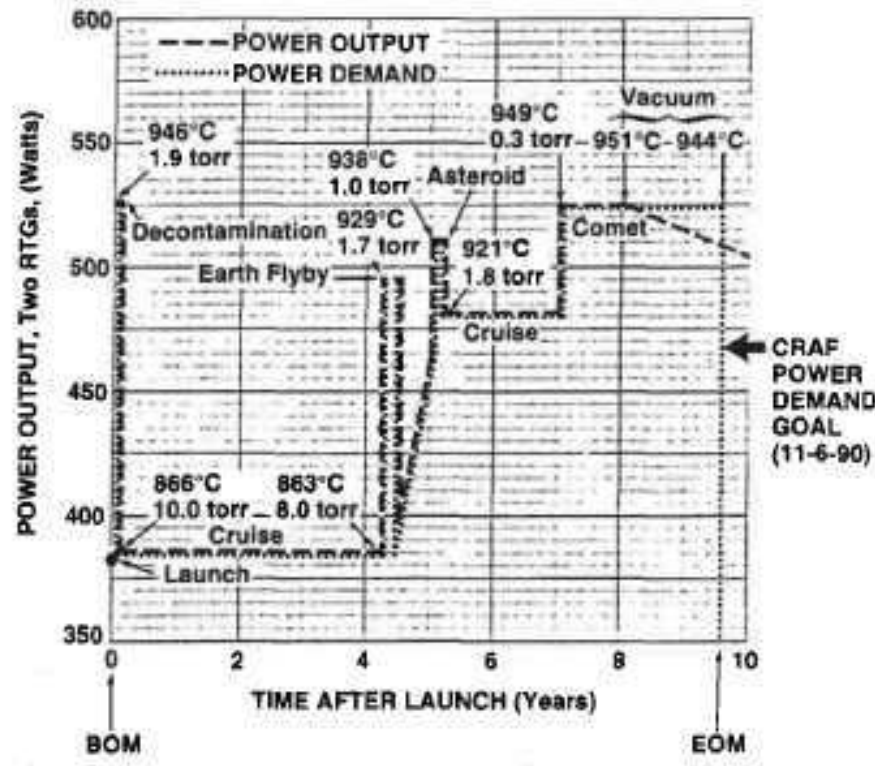

As can be seen, for the 36-ring RTG the power output matches the power demand for the first 8.2 years of the mission. At that time the required helium pressure drops to zero. After that time the RTGs are evacuated and their power output drops below the demand schedule. At the end of mission, the power output is 14 watts below the EOM demand.

Figure 32. Effect of Controlled-Rate Venting of Helium on Output of 32-Ring RTGs

$35^{\circ}$ Separation, 18 Heat Source Modules, 245-Want BOM, 3" Fins)

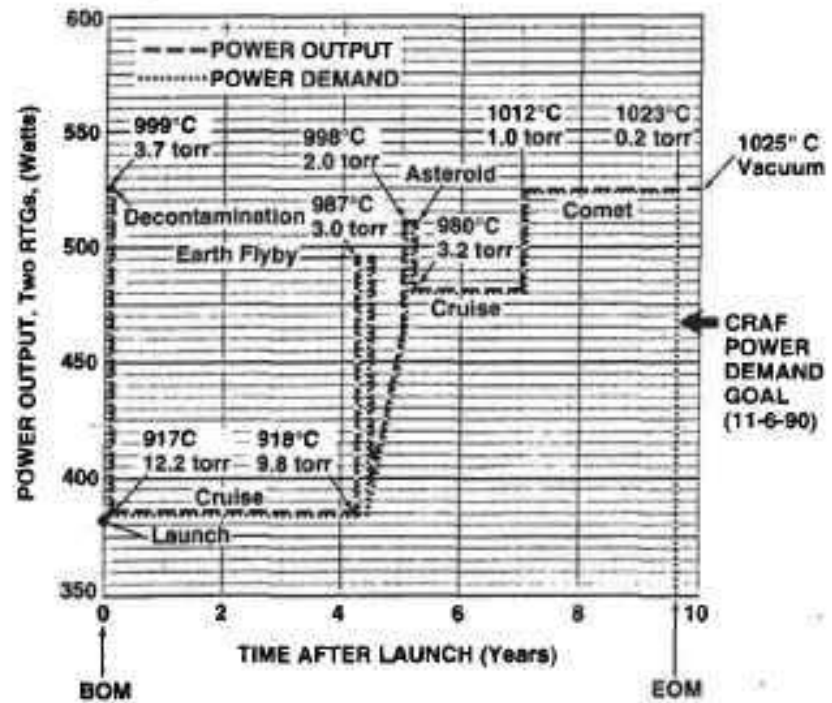

For the 32-ring RTG, Figure 32 shows that the power output and demand are matched throughout the mission. But the required helium pressure during the comet science phase is extremely low $0.2<\mathrm{p}<1$ torr).

\section{RECOMMENDATION}

As can be seen, the controlled-rate helium-venting stratagem results in the lowest thermoelectric degradation, and - when coupled with reduced number of couple rings - can actually match the prescribed power demand schedule throughout the mission. Nevertheless, it was not recommended by Fairchild, because the required accurate control of the very low helium pressure shown was deemed to be impractical. The author's preference was for a combination of voltage switching, increased fin size, and 34 instead of 36 couple rings. This combination was deemed to be practical, and close enough to the previously flown Galileo and Ulysses design not to require new safety or qualification tests. Therefore, it was judged by the author that the above revisions could be implemented in time for the CRAF and Cassini missions.

The combined effectiveness of the above modifications relative to the CRAF power demand goals is displayed in Figure 33. The output of two standard GPHS RTGs is shown by the dashed curve, and the output of two modified RTGs (with voltage switching) is shown by the dotted curve.

Figure 33. Comparison of Standard and Modifled RTGs (35 Separation, 18 Heat source Modules, 245-Watt BOM)

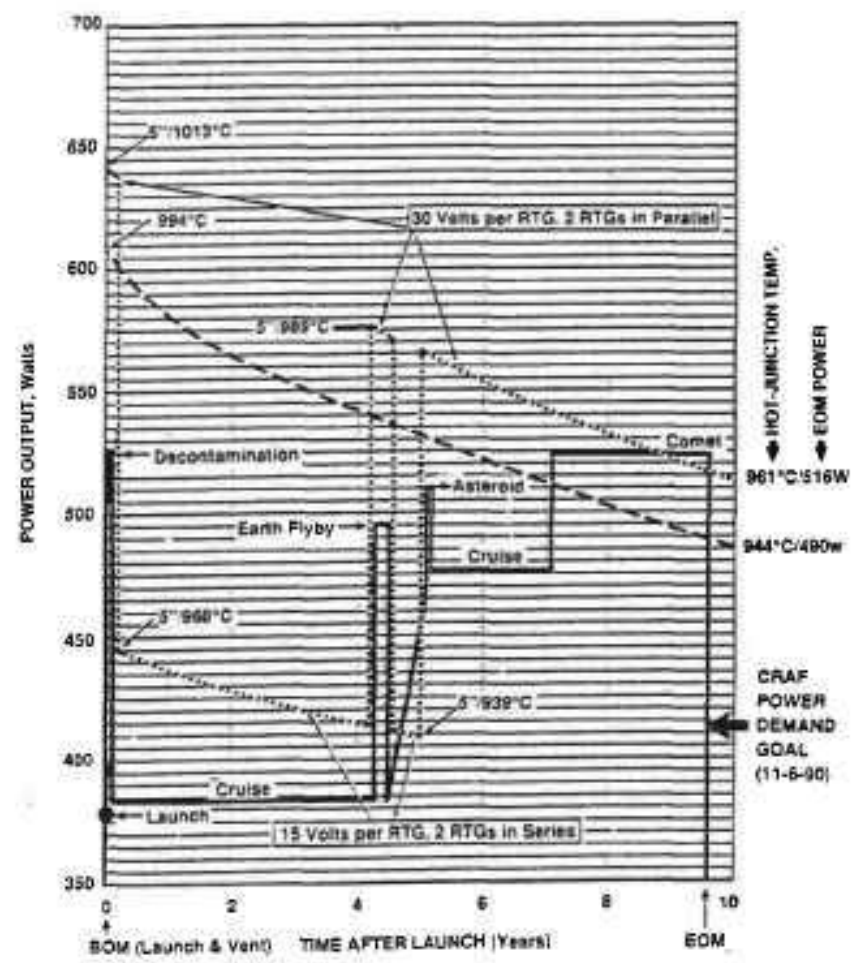


As can be seen, the latter has an EOM output 26 watts above that of the standard RTGs, though still 8 watts below the EOM goal. Except for this shortfall at the end of the mission, which would have to be accommodated by a small load reduction, the modified RTGs meet all of the mission's power demand goals.

\section{JPL DECISION AND STUDY CONCLUSIONS}

Although the above two-RTG option could have resulted in substantial time and cost savings for the CRAF project, the JPL Project Office ultimately decided against its use. This was primarily because the project's science group wanted greater power margin to accommodate anticipated increases in power demand (above the present contingency allowances), and because the CRAF power demand schedule shown in Figure 33 assumes a good deal of load switching to reduce power demand peaks. Such load switching is undesirable, because of potential reliability problems. It was anticipated that the use of three RTGs for the CRAF mission would minimize the need for load switching. Also, addition of the third ATG was expected to permit elimination of the batteries.

After JPL's decision to employ three RTGs per spacecraft, the detailed thermal and electrical analysis of the obstructed RTGs had to be repeated for the three-ATG case. The mounting of the RTGs on the Mariner Mark-2 spacecraft is illustrated in Figure 34. JPL specified a separation angle of 34 degrees between neighboring RTGs. In this configuration, the middle RTG will run hottest (and degrade fastest) because it is blocked on both sides.

Figure 34. Mounting of Three RTGs on Mariner Mark-2 Spacecraft (34. Separation Between Neighbors)

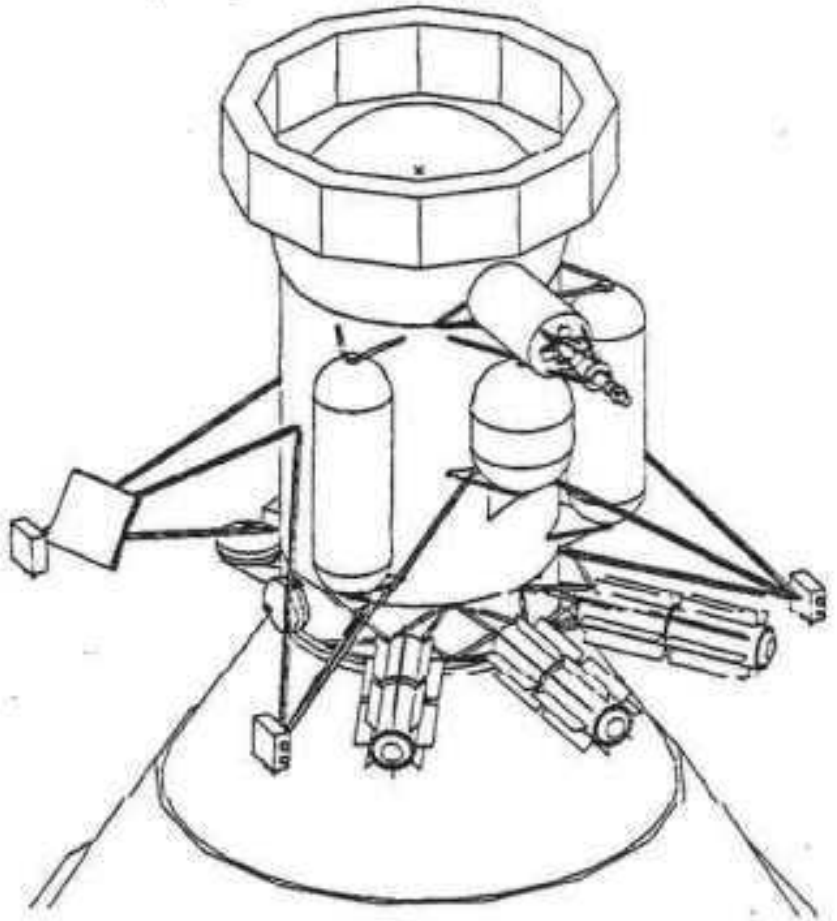

The coupled thermal and electrical analysis of the three-RTG configuration was carried out for BOM thermal powers ranging from 225 to 255 watts per heat source module. The analyses assumed a typical three-year interval between chemical separation (BOL) and launch (BOM). The computed power histories of the three RTGs are shown parametrically in Figure 35. Each curve shows the fuel enrichment at BOL, the thermal power per heat source module and maximum hot-junction temperature at BOM, and the power output at EOM. The curves shown extend for 12 years, covering both the CRAF and Cassini mission durations.

Figure 35. Effect of BOM Thermal Power on Output History of Three Std. RTGs

(34 Between Neighboring RTGs, 18 Heat Source Modules per RTG)

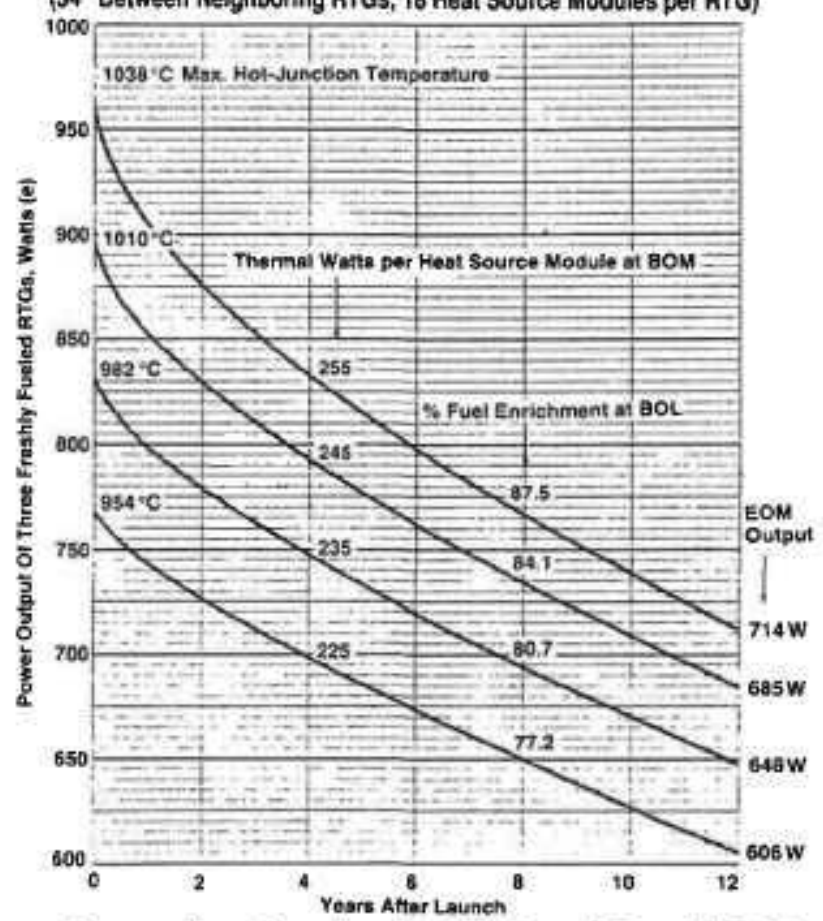

As mentioned earlier, the CRAF and Cassini RTGs will be fueled entirely from existing inventory. For the earlier case of five RTGs ( 3 for Cassini and 2 for CRAF), selection of the highest-enrichment fuel yielded a BOM thermal power of 241 watts per heat source module. For the six-RTG case selected by JPL, it is necessary to include some lower-enrichment fuel, which drops the BOM thermal power of the freshly fueled ATGs to 238 watts per heat source module.

The corresponding power output histories for the two missions are displayed in Figures 36 and 37 . Each figure shows two power output curves, representing two fueling options. The solid curve represents the preferred option, in which three freshly fueled RTGs are used for the Cassini mission while the CRAF mission employs two freshly fueled units and one aged spare (F5) left over from the Galileo/Ulysses program. This option is preferred because it favors the Cassini 
mission with its higher power demand. The second curve represents a back-up option, in which the lowerpowered aged RTG is used for Cassini instead of CRAF. This is clearly less desirable, and would only be done if the three freshly fueled RTGs were not available in time for the Cassini launch.

Figure 36. CRAF POwer Supply versus Demand (34: Separation Between Neighboring RTGs)

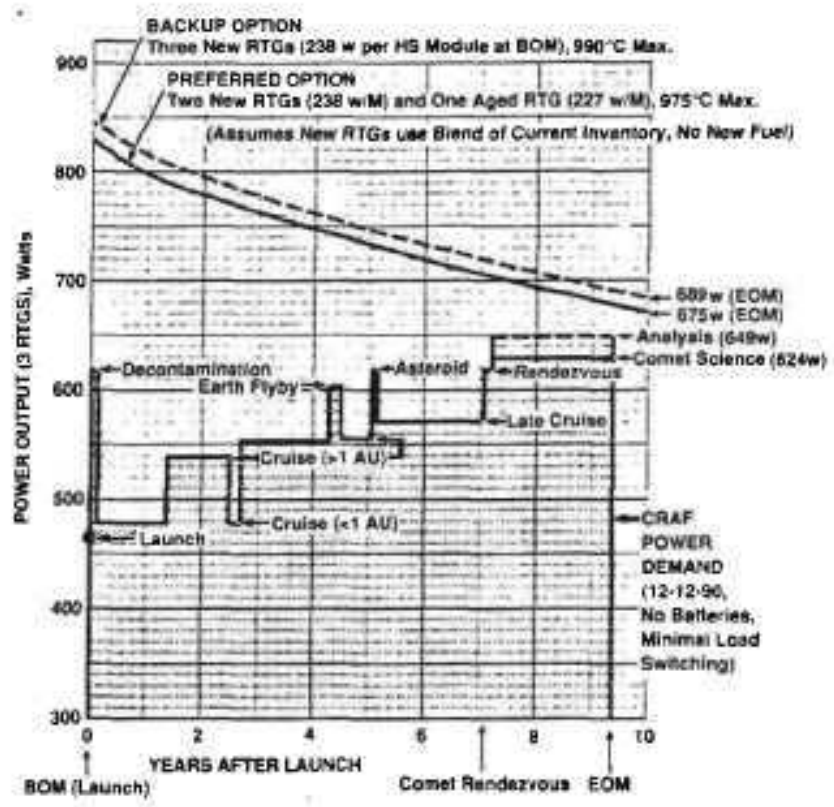

Figure 37. CASSINI Power Supply versus Demand (34 $4^{\circ}$ Separation Between Neighboring ATGs)

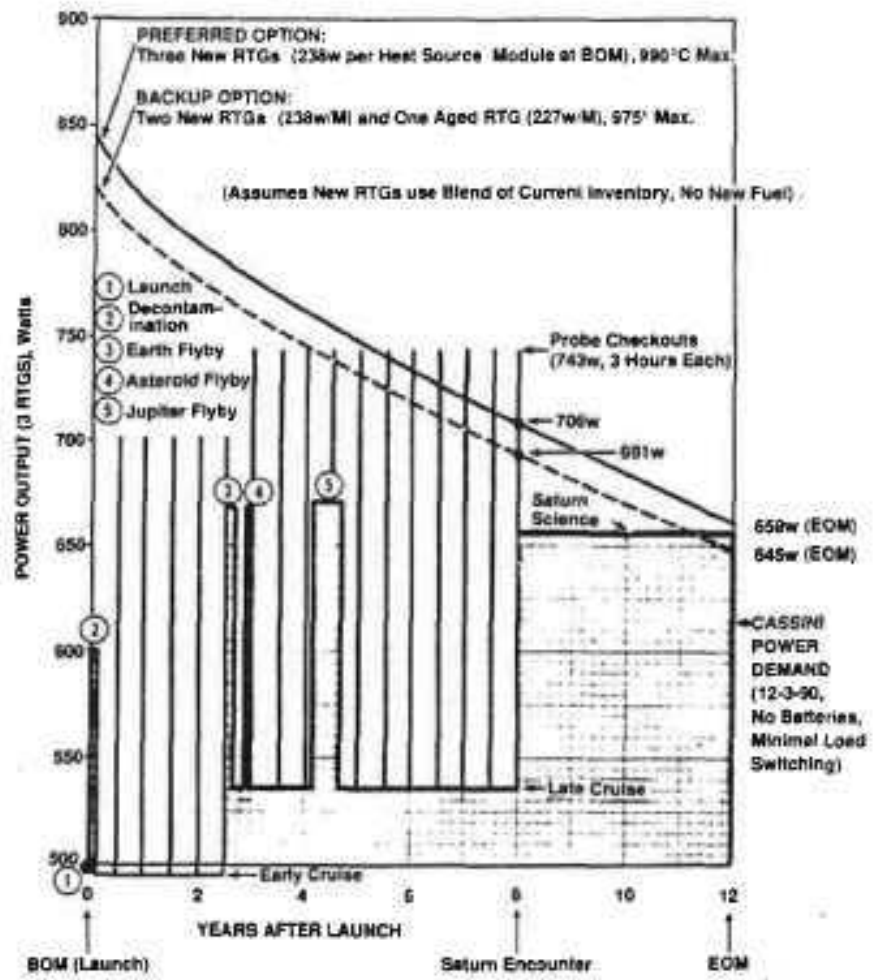

Figure 36 shows that either option satisfied the JPL-specified power demand goal for CRAF; and Figure 37 shows that the preferred option satisfies all of the specified Cassini goals except for some of the semi-annual 3-hour probe check-outs shortly before Saturn encounter. As can be seen, the supply fell short of meeting the demand during the last five checkouts for the preferred option, and the last eight checkouts for the back-up option. These shortialls would have to be made up by JPL mission planners, e.g. by briefly shutting off non-essential loads during the last few probe check-outs.

The power supply curves shown in Figures 36 and 37 represent the as-computed results of the thermal and electrical analyses, without any reductions for contingencies. They represent the author's best estimates, but presumably exceed the power profile to which DOE and its system contractor (GE) would be willing to commit themselves. Even though standard GPHS RTGs have already been built, tested, and successfully flown in space, and the present prediction methods have been validated against measured performance data, the new generators may not quite match the output of the previously built units. DOE has not yet decided what performance level it is willing to commit to, but Figures 38 and 39 illustrate the effect of hypothetical $5 \%$ and $10 \%$ reductions on meeting the power demand goals of the two missions (with the preferred launch sequence). Figure 38 shows that all the CRAF goals can be met with a $3 \%$ contingency reserve, but not with at $5 \%$ reserve.

Figure 38. CRAF RTG Power Margin

Power Supply

3 ATGs, 34. Separation Between Nelghbors

2 ATGe are Freanhy Fueled

Uniform Blend of Current inventory, 81. T\%. Pu-238 Errichment at Separation, Same Fuel Denaity an in Gollleo RTOe, 4284 Thermat Watts per RTG at BOM

1 RTS is 13-Yoar-Old Gallloo Spare: 4086 Thermal Watse a: BOM

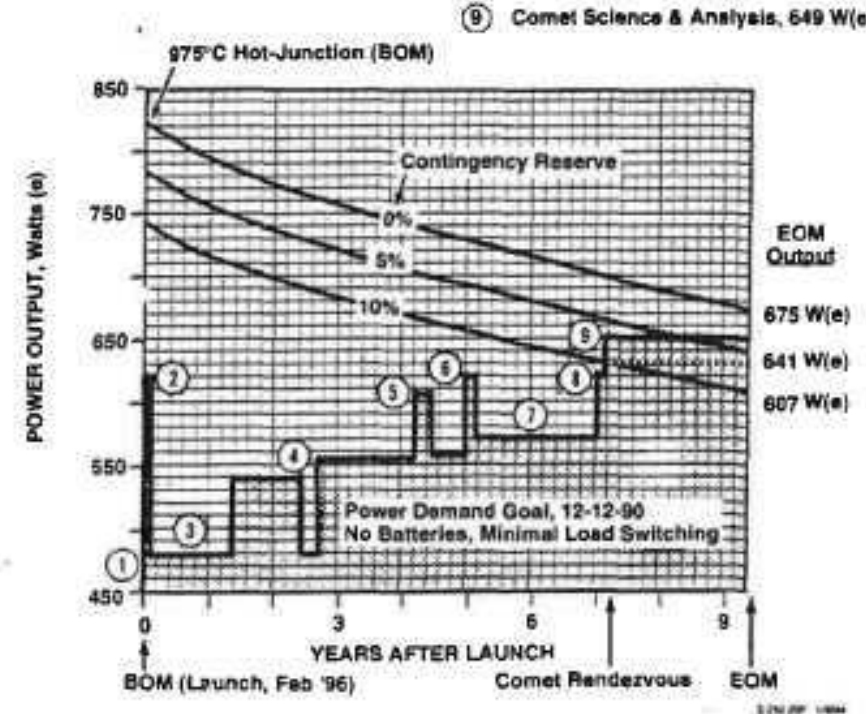

Power Demand

(1) Launch, $459 \mathrm{~W}(a)$

(2) Decontamination, $819 \mathrm{~W}(\boldsymbol{e})$

(3) Earny Crulse (e $1 \mathrm{AU}), 47 \mathrm{~W}$ W(s)

(4) Enrly Crulse (> 1 AU) 599 W(e)

(5) Earth Flyby, 603 W(t)

(6) Astereid Flyby, $619 \mathrm{~W}(\mathrm{e})$

(7) Late Cruise, $571 \mathrm{~W}(\mathrm{e})$

(9) Rendervous, $619 \mathrm{~W}(0)$

$9750 \mathrm{C}$ Hot-dunction (BOA) 
Figure 39. CASSINI RTG Power Margin

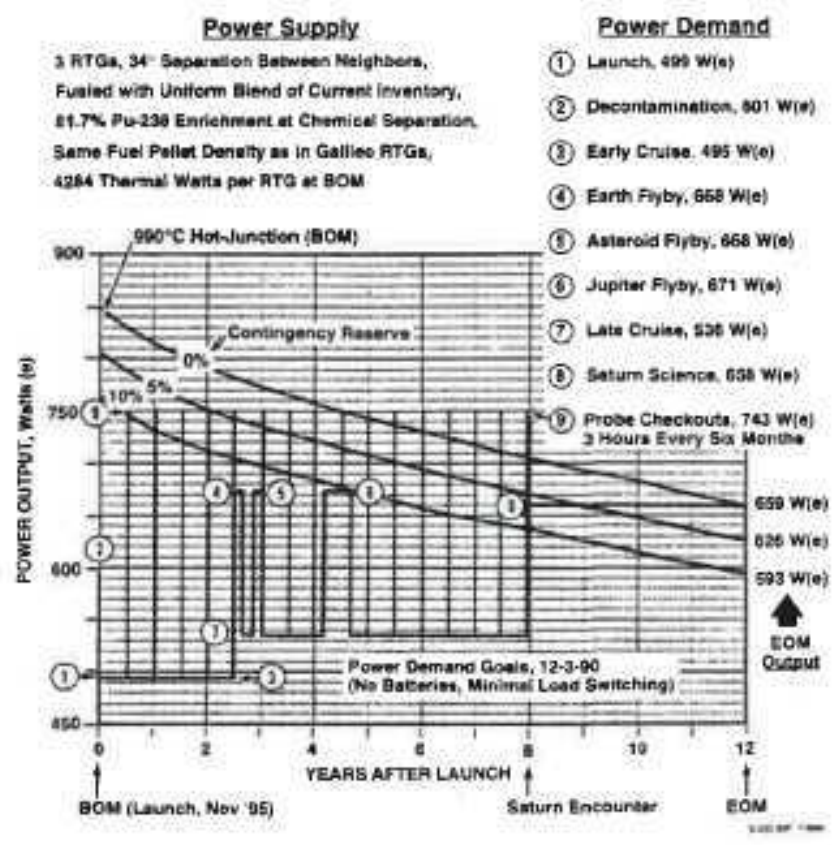

Figure 39 shows that for the Cassini mission the predicted EOM just matches the power requirement for the Saturn Science segment of the mission. Therefore, shortfalls would require additional load switching or other reductions toward the end of the mission.

The description of the various power enhancement stratagems investigated and of their computed effectiveness was documented in detail in this paper to make the information available in case of future power enhancement requirements.

\section{ACKNOWLEDGMENT}

The above-described work was supported by the U.S. Department of Energy's Office of Special Applications, R. Lange, Director, and E. Mastal, Deputy Director.

\section{REFERENCES}

[1] Draper, A.F (1988). The Meriner Mark II Program." Proe, of AlAA Conlerence, hold in Reno, Nevada, January 1906, M880067

[2] Schoek, A., and H. Sookiazian $(1979)$ "Conceptual Design of the ATG for Solar-Polar Miasion,- Presented at the 14th Intersociety Energy Conversion Engineering Conterence, heid in Boston, Massachusetts, August 1979, 1799307.

[9] Cockfield, A.D., A.F. Hartman, C.D. Kelly (1960) "RTG Power Sources for International Solar Polar Misaion," Proc, of the 15th Intersociety Energy Conversion Engineering Conference, hald in Seattie, Washington, 1980.

[4] Cockfieid, R.D. (1996) 'Qualification of GPHS-RTG for Gsilleo and Ulysses," Proc, of the 21 si Intersociety Energy. Conversion Engineering Conletence, held in San Diego, Cenlfornia, 1986 .

[5] Schock, A. (1980) -Design Evolution and Verification of the General Purpose Heat Source". Vol, 2, pages 1032-1042. Proc. of the 15th Intersociety Energy Conversion Engineering Conference, hold in Seattle, Washington, 1980.

[6] Jolfe, A. F. (1957) Semiconductor Thermoeiements and Thermoelectric Cooling inforsearch, London, 1057.

[7] Schoek, A., C. T, Or, and E, A. Skrabek (1990) "Thermal and Electrical Anniysis of Mars Rover RTGs, and Performance Comparinon of Alternative Deaign Options," Trans, of the Seventh Symposium on Space Nuclear Power Systems, held in Albuquerque. Now Mexico, January 1990.

[B] Schock, A. T. Hamrick, and K. Sankarankandath (1990) "Design and Structural Analysis of Mars Rover RTG."Trans, of the Seventh Symposium on Space Nuclear Power Systoms. held in Albuquerque. Now Moxico, Jamusry 1090

[9] Schock, A, ot al, (1999) "Mars Rover RTO Study, FSC-ESD. 217/89/450A. Fairchlid Space Company, Germantown, Maryland, November 1989.

[10] Loffreda, J. (1982) Engineoring Unit Test Results," PIR-6377. General Electric, Spsee Division, Philadeiphia, Pennsylvania, May 1982.

[11] ITAS: Integrated Thermal Analysis Systems, Analytix Corporation, Timonium, Maryland, 1990.

[12] Gaski, J, (1986) SINDA (System Improved Numerical Differencing Analyzet) version 1.23 from Network Analyeis Associate, Fountain Valloy, Californin.

[13] Raag, V. and K.P. McCarron (1973) "Mathematical Model and Computer Program for the Design and Analysis of Silicon. Germanium Air-Vac ATG Degradation," Memorandum \#15. Syncal Corporation, Sunnyvale, Callfornia, October 1973.

[14] Mowery, A.L. (1975) "Preliminary Power Degradation Report,Interollice Memorandum to J. Lombardo, U.S. Energy Research and Development Administration, Weshington, D.C.. May 1975.

[15] Jet Propulsion Laboratory (1989), D-3384, -Mariner Mark II, Comet Rendezvous and Asterold Flyby and Cassinl, Technical Definition and Cost Review", Volume 1. May 23, 1989.

[16] Eck, Marshail (1990) *CRAF/Cassint Contingency Plans Using Only Exting Pu ${ }^{238}$ Inventory", Fairchild Space, FSC-217-90. $478 B$, October 25, 1990 .

[17] EGsG Mound Applied Technologies (1990) "Heat Source and RTG Status Report', Miamisburg, OH, November 1990. 